\title{
Directional response of structures to thunderstorm outflows
}

\author{
Stefano Brusco • Valentino Lerzo • Giovanni Solari 1
}

Received: 23 January 2019/ Accepted: 19 April 2019/Published online: 14 May 2019

(C) The Author(s) 2019

\begin{abstract}
Due to the combined effect of the background wind, the translation of the downburst and the radial nature of the downdraft after the touchdown, a peculiar characteristic of a thunderstorm outflow as detected by an anemometer or experienced by a structure is the potential variation of the flow direction. The study of the wind-excited response of structures has habitually neglected this issue because, dealing traditionally with synoptic events, their direction is endowed with a regular pattern. This paper proposes two families of methods to take into account directional changes in the evaluation of the windexcited response of structures. The first one generalizes the method usually applied to downbursts, which by its nature implicitly assumes a non-directional response. The second one is based on a new directional decomposition strategy of the wind speed that represents a generalization to thunderstorm outflows of what is historically done for synoptic events. The conceptual aspects involved in and the results provided by these methods are critically discussed and
\end{abstract}

S. Brusco · V. Lerzo · G. Solari $(\bowtie)$

Department of Civil, Chemical and Environmental

Engineering, Polytechnic School, University of Genoa,

Genoa, Italy

e-mail: stefano.brusco@edu.unige.it

V. Lerzo

e-mail: s3547579@studenti.unige.it

G. Solari

e-mail: giovanni.solari@unige.it compared both to each other and with regard to the traditional non-directional structural analysis of thunderstorm outflows.

Keywords Aerodynamic loading - Directional analysis - Synoptic wind - Thunderstorm outflow · Wind-excited response

\section{Introduction}

The study of the dynamic response of structures to synoptic winds usually regards the wind loading effects, first the displacement and the probability density function (PDF) of its maximum value. To pursue this aim, the wind velocity is first decomposed into two components: the mean wind velocity, in a period between $10 \mathrm{~min}$ and $1 \mathrm{~h}$ [1], with constant magnitude and direction, and the residual fluctuation, dealt with as a stationary Gaussian process expressed in terms of a longitudinal and a lateral turbulence component. This procedure, which represents the first ring of the Davenport Chain [2], facilitates the classical analysis of the structural behaviour in terms of alongwind and crosswind response (e.g. [3]).

The study of the dynamic response of structures to thunderstorm outflows has followed a different pathway $[4,5]$, since the horizontal component of the resultant wind velocity is usually decomposed into a 
slowly varying mean wind velocity and a residual nonstationary fluctuation [6, 7]. Many efforts have been devoted on how best performing this separation, adopting procedures involving wavelets, empirical mode decomposition and kernel regression [8-11] or, more easily, a moving average filter $[12,13]$. On the other hand, no discussion has been carried out on the quantitative role of the changes of the wind direction due to combining the background wind, the translation of the downburst and the radial nature of the downdraft after the touchdown. Though many authors discussed this aspect qualitatively [7, 14, 15], pointing out the relevant character of this phenomenon, no model of the thunderstorm outflow takes into account this phenomenon in explicit form. This fact also precludes any chance to separate turbulence into a longitudinal and a lateral component as usually done for synoptic events.

This attitude, by now a custom or a sort of axiom in thunderstorm modelling, unavoidably led to some major shortcomings. First, it caused a deep difference between the study of the dynamic response to synoptic winds and to thunderstorm outflows, making questionable any comparison. Second, it oriented the literature on the dynamic response of structures to thunderstorm outflows towards the implicit assumption that the response occurred in an alongwind invariant direction, independently of whether analyses were carried out in the time-domain [12, 16-20], by means of the evolutionary power spectral density method [17, 21-23], or using the response spectrum technique [20, 24, 25]; this precluded any chance to separate the alongwind response from the crosswind one, as it is classical for synoptic winds. Third, it did not allow to take into account the variation of the angle of attack jointly due to the turbulent fluctuations and to the changes of the wind direction. A noteworthy exception to this trend is represented by Ref. [26], where a numerical model has been implemented that takes into account the translation of the thunderstorm cell with respect to the structure, this making the structure subjected to a couple of horizontal orthogonal forces applied along its principal axes in plan. Nevertheless, this approach missed to define a traditional couple of the longitudinal and lateral turbulence components, of the alongwind and crosswind forces, and of the alongwind and crosswind response.

To overcome these shortcomings, a new directional decomposition strategy of the wind speed has been recently formulated [27] which opens the doors to robust comparisons and parallel analyses of thunderstorm outflows and synoptic wind in terms of wind speed, of wind loading and of structural response, taking into account the time-variation of the slowlyvarying mean wind speed and direction. Besides the effects of the change of direction of thunderstorm outflows in the wind loading and response of structures, a clear evaluation of this parameter is fundamental to recognize the travelling nature of the downbursts, to reconstruct their evolution and to clarify the crucial role of the intensification of the wind speed due to the translation of the thunderstorm cell.

This paper focuses on the role of the change of direction in thunderstorm outflows with regard to the structural response. This aim is pursued by making recourse to the dataset of the records acquired during the "Wind and Ports" [28] and "Wind, Ports and Sea" [29] European projects and by evaluating the windexcited response of two vertical slender test structures in the time domain $[19,20]$. The role of the wind direction is considered since the wind speed decomposition stage [27], by means of two families of methods, which are afterward described. Based on them, the time-varying aerodynamic forces are analytically expressed through the classical quasi-steady theory, generalized here by the use of aerodynamic coefficients slowly-varying over time.

Section 2 describes two real structures and ten thunderstorm outflow records used to investigate the issue dealt with. Section 3 illustrates the classical and the new directional decomposition of the wind velocity and direction. Section 4 reconstructs wind fields compatible with the chosen structures and thunderstorms records; the equivalent wind spectrum technique [30, 31] drastically simplifies such reconstruction. Section 5 defines different criteria, coherent with the decomposition rules introduced in Sect. 3, through which the aerodynamic wind loading is evaluated. Section 6 describes the equations of motion of the wind-excited response and the obtained results. Section 7 summarizes the main conclusions and draws some prospects regarding the discussed topic. 


\section{Test cases}

This section describes two real test structures (Sect. 2.1) and ten real thunderstorm outflow test records (Sect. 2.2) used hereinafter to study the problems dealt with in this paper. This section anticipates the following theoretical Sects. 3-5 and in part Sect. 6 not just because theory is limited to the test cases discussed here, but because they are used below to exemplify the application of some theoretical developments. With the same motivations, this principle was previously used in $[19,20]$.

\subsection{Structures}

With the aim of focusing the analysis on thunderstorm outflow directional effects, two real slender vertical structures with comparable heights are examined in this paper as reference test cases: a steel telecommunication antenna mast in the port of La Spezia (S1) and the Endless Column (S2) conceived by Costantin Brâncuşi (1876-1957). Both structures are low/ medium-rise ones in order to exalt thunderstorm effects.

The antenna mast [19] is made up of two shafts with tubular circular section and total height $36 \mathrm{~m}$. The bottom shaft is $30 \mathrm{~m}$ long and consists of 5 parts, each one of them $6 \mathrm{~m}$ long, with constant outer diameters 914.8, 812.8, 711.2, 609.6, 508.0 $\mathrm{mm}$ and thicknesses 8.0, 8.0, 7.1, 6.3, $5.6 \mathrm{~mm}$, respectively. The upper shaft is put above the previous one and is characterized by a $6 \mathrm{~m}$ length, constant outer diameter $193.7 \mathrm{~mm}$ and thickness $7.1 \mathrm{~mm}$; it is predisposed to carry up to 6 antennas that are not present in this configuration.

The Endless Column [32] is located in the Constantin Brâncuşi's Sculptural Ensemble at Târgu Jiu, Romania. The column is the ensemble of two parts: an internal steel spine with square cross-section and an external assemblage of cast-iron modules. The spine's cross-section varies from the base to the top (Fig. 1a). It is filled with concrete up to the $5.50 \mathrm{~m}$ height. The external coating consists of the superimposition of 15 complete modules, plus one half at the base and one half at the top; each module consists of two trunks of a square base pyramid joined in correspondence of the largest bases (Fig. 1b). The total height of the column is $29.26 \mathrm{~m}$ and its slenderness ratio is 66 .

Figure 2 shows a picture and the model scheme for both structures. Table 1 shows their main properties:
$H$ is the height; $n_{1}, \xi_{1}, m_{1}$ are respectively the natural frequency, the structural damping coefficient and the modal mass of the first mode of vibration; $\zeta_{1}$ is the exponent of the power law that best approximates the first modal shape, $\psi_{1}(z)=(z / H)^{\zeta_{1}} ; N$ is the number of joints of each structural model in correspondence of which the wind-loading time-histories are applied and the structural response is determined. The choice of examining two structures of limited height was made with the aim of enhancing the actions of thunderstorm outflows and reducing the role of the shape of their velocity profiles $[19,20]$.

In general terms, the aerodynamic coefficients of a structure in a flow field mainly depend on the angle of attack $\theta$ and on the Reynolds number $R e$.

$\mathrm{S} 1$ is considered here as a polar-symmetric structure. This reduces the double dependence of its aerodynamic coefficients to the sole Reynolds number. This latter quantity has been determined according to the Italian Guide on Wind actions and effects on structures [33].

As far as S2 is concerned, its aerodynamic coefficients have been evaluated through wind tunnel tests [32] according to which the Reynolds number plays a marginal role. This reduces the double dependence of its aerodynamic coefficients to the sole angle of attack. Figure 3 shows the measured drag and lift coefficients, $c_{d}, c_{l}$, and $c_{d}+c_{l}^{\prime}, c_{d}^{\prime}-c_{l}, c_{d}^{\prime}$ and $c_{l}^{\prime}$ being the angular prime derivatives of $c_{d}$ and $c_{l}$. All these quantities have been normalised with regard to the side of the largest base, $b=90 \mathrm{~cm}$, of the pyramidal trunks that make up the modules (Fig. 1b).

The choice of examining two structures with polarsymmetric (S1) and square (S2) cross-section was made with the aim of mitigating the effects of the changes in wind speed direction for the former and to enhance them for the latter.

Figure 4 shows the $X$ and $Y$ axes of the Cartesian reference system adopted herein, in the plan of the structure cross-section, $Z$ being vertical and directed upwards. For both the test structures $X$ and $Y$ are principal axes of inertia and axes of symmetry too.

\subsection{Wind events}

In order to investigate the directional response of the two test structures described above to a representative set of thunderstorm outflows, ten events have been 

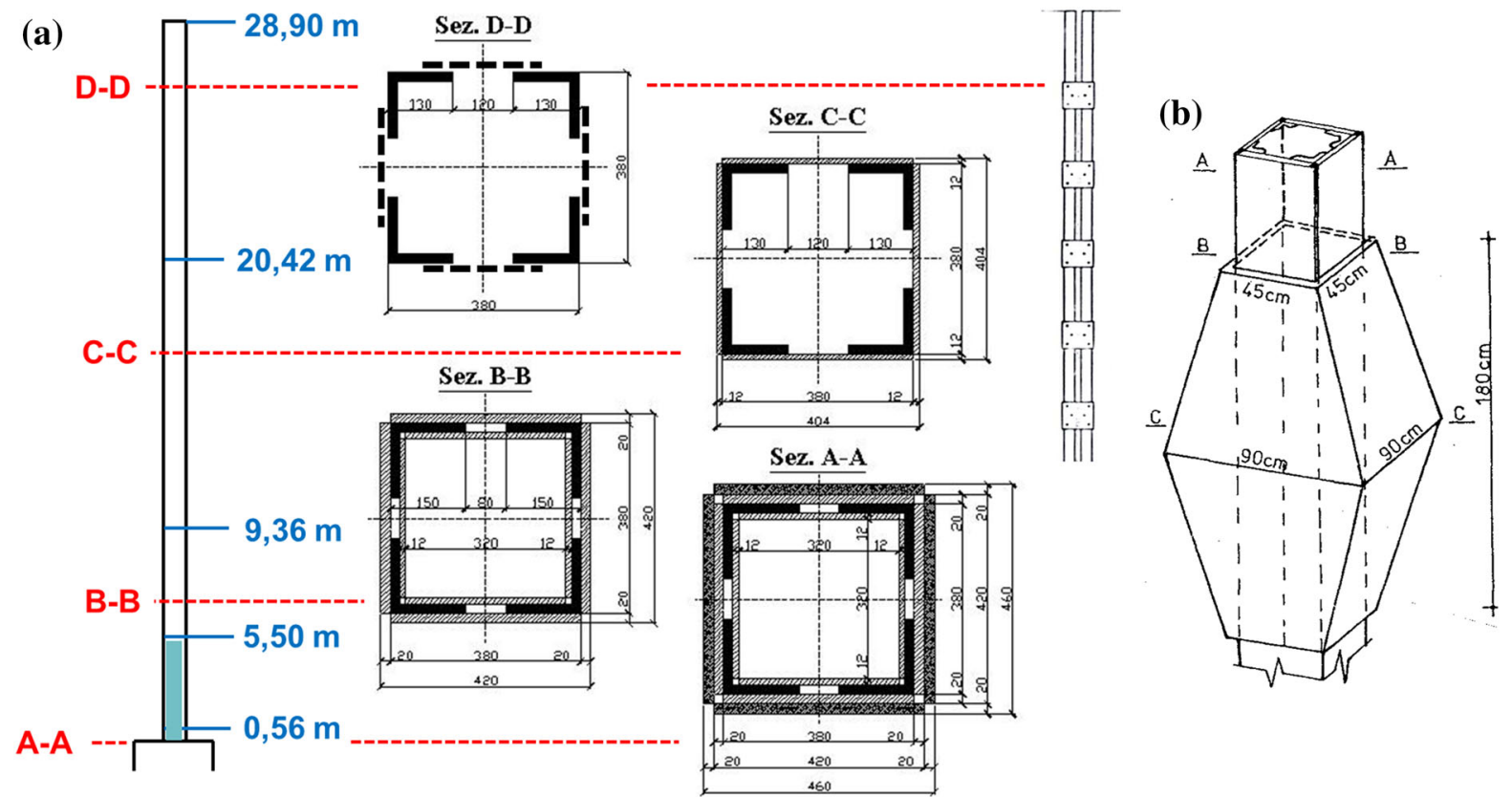

Fig. 1 Brâncuşi’s Endless Column: a steel spine; b coating modules

(a)

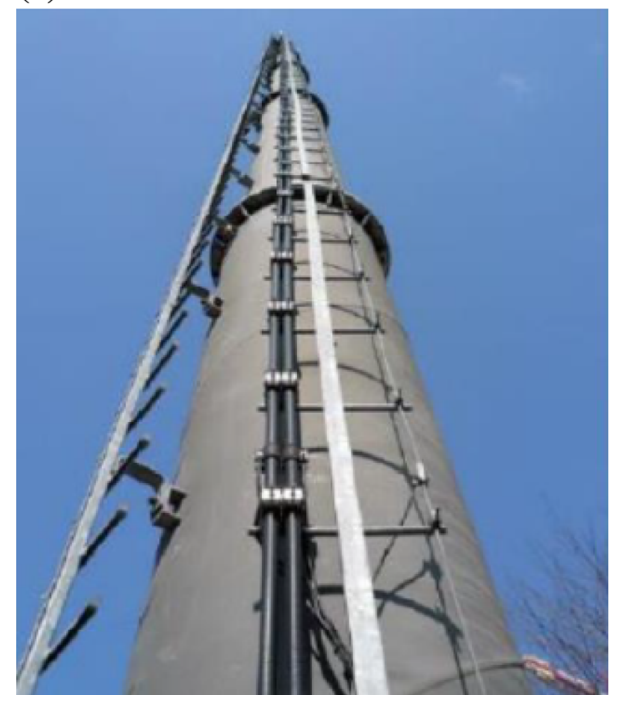

(b)

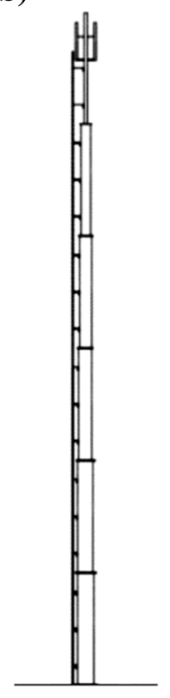

(c)

(d)

Fig. 2 Pictures and schemes of the structure test cases: a, b S1; c, d S2

Table 1 Main properties of the two structure test cases

\begin{tabular}{llllrlr}
\hline Structure & $H(\mathrm{~m})$ & $n_{1}(\mathrm{~Hz})$ & $\xi_{1}(-)$ & $m_{1}$ & $\zeta_{1}(-)$ & $N$ \\
\hline S1 & 36.00 & 0.8209 & $1.00 \%$ & 412 & 2.15 & 19 \\
S2 & 29.26 & 0.5130 & $1.93 \%$ & 4618 & 1.75 & 6
\end{tabular}

selected from the "Wind and Ports" (WP) [28] and "Wind, Ports and Sea" (WPS) [29] projects database. They are called, hereinafter, as WE1-WE10. Table 2 shows their main properties. Appendix A provides the wind speed and direction diagrams of these events. All of them are defined over the time interval $\Delta T=10 \mathrm{~min}$. The following analyses conventionally 
Fig. 3 Aerodynamic coefficients of S2: a drag coefficient, b lift coefficient; coefficients $\mathbf{c} c_{d}+c_{l}^{\prime}$ and $\mathbf{d}$ $c_{d}^{\prime}-c_{l}$

Fig. 4 Reference system for $\mathbf{a} \mathrm{S} 1$ and $\mathbf{b} \mathrm{S} 2$
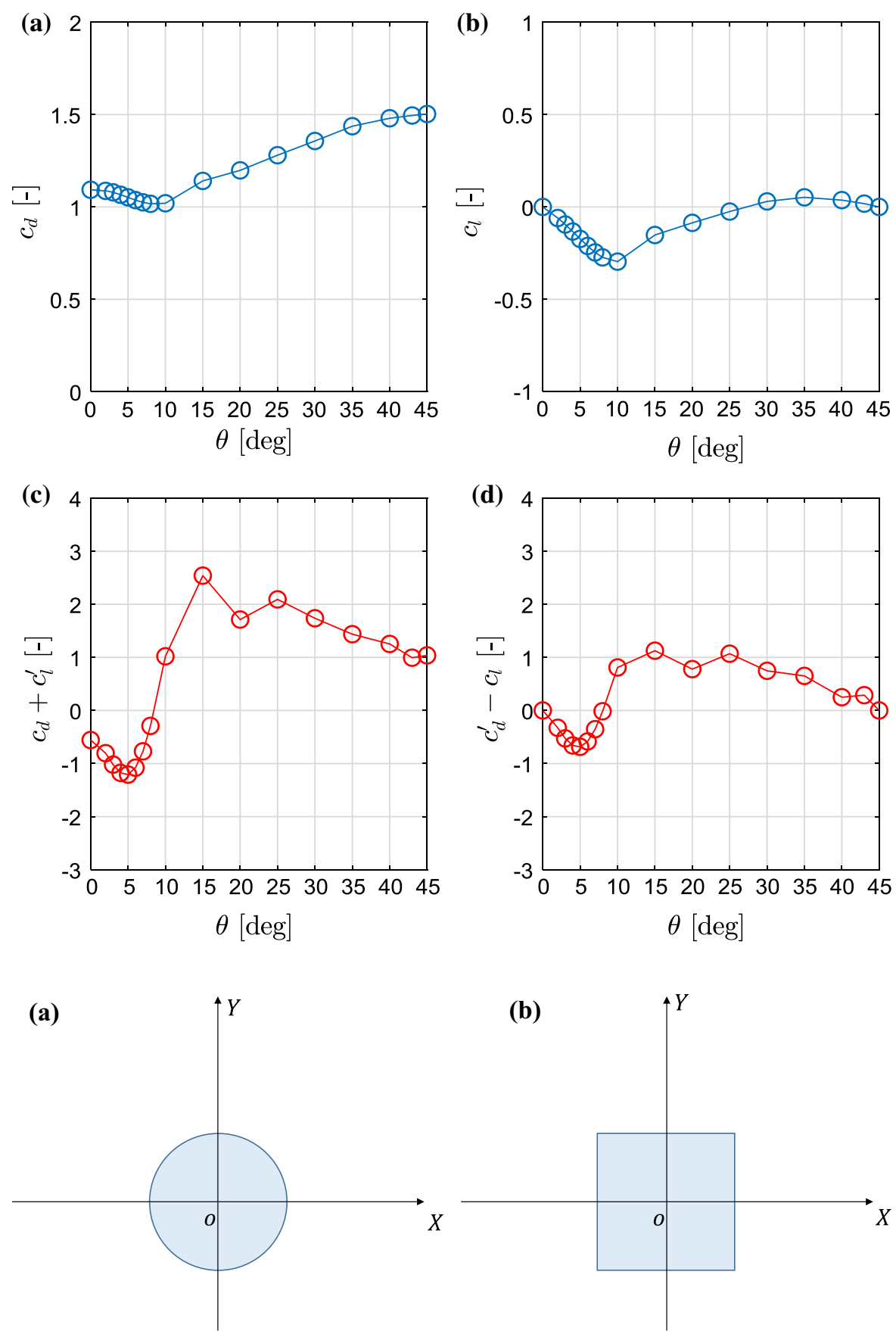

assume that the $X$ and $Y$ axes in Fig. 4 are directed respectively along the $\mathrm{W}-\mathrm{E}$ and $\mathrm{S}-\mathrm{N}$ directions.

Sections 3, 4 and 6.1 focus on the first of these events, WE1, as the reference test-case. Section 6.2 provides and discusses the whole of the results concerning the ten events WE1-WE10.

\section{Wind speed decomposition}

Focusing on the horizontal component of the wind speed, the anemometric data is usually stored in terms of components $\left(V_{X}, V_{Y}\right)$, being $V_{X}$ directed from West to East and $V_{Y}$ from South to North. 
Table 2 Generalities about the ten thunderstorm outflow records selected from the WP and WPS database

\begin{tabular}{lllll}
\hline Event name & Location, Port of & Anemometer no. & Date & 1-s peak $(\mathrm{m} / \mathrm{s})$ \\
\hline WE1 & La Spezia & 2 & $30 / 01 / 2015$ & 19.14 \\
WE2 & Genoa & 1 & $28 / 10 / 2012$ & 25.78 \\
WE3 & Genoa & 1 & $03 / 11 / 2012$ & 17.50 \\
WE4 & Livorno & 1 & $16 / 12 / 2011$ & 33.64 \\
WE5 & Livorno & 4 & $18 / 01 / 2014$ & 18.34 \\
WE6 & Livorno & 5 & $16 / 11 / 2010$ & 27.63 \\
WE7 & Livorno & 5 & $15 / 10 / 2012$ & 22.57 \\
WE8 & La Spezia & 3 & $25 / 10 / 2011$ & 33.98 \\
WE9 & La Spezia & 3 & $15 / 10 / 2012$ & 22.53 \\
WE10 & La Spezia & 3 & $09 / 02 / 2014$ & 21.55 \\
\hline
\end{tabular}

In the case of synoptic winds, the mean wind velocity and its direction are first evaluated; the residual stationary fluctuation is then separated into a longitudinal and a lateral turbulence component. This operation is functional to separate the mean static part and the dynamic part of the structural response, the latter one being usually dealt with in terms of an alongwind and a crosswind response.

A different approach has been traditionally applied to thunderstorm outflows, for which the resultant wind velocity is decomposed into a slowly-varying mean part and a residual non-stationary fluctuation. The wind direction is usually regarded from the sole qualitative viewpoint and the structural response is implicitly assumed in the alongwind invariant direction (Sect. 3.1).

Having two different philosophies of decomposition, though, precludes a parallel treatment and a robust comparison between the structural response to thunderstorm outflows and to synoptic winds. This shortcoming was overcome in [27], where a unitary directional decomposition was introduced (Sect. 3.2).

\subsection{Classical decomposition}

This approach [6, 7] consists of decomposing the resultant velocity $U$ :

$U(t)=\sqrt{V_{X}^{2}(t)+V_{Y}^{2}(t)}$

into a slowly-varying mean velocity $\bar{U}$ and a fluctuation $U^{\prime}$ that is later expressed as the product of the slowly-varying standard deviation $\sigma_{U}$ by a reduced turbulent fluctuation $\tilde{U}^{\prime}$ dealt with as a rapidly-varying stationary Gaussian random process with zero mean and unit standard deviation. So, the resultant velocity may be expressed as:

$$
\begin{aligned}
U(t) & =\bar{U}(t)+U^{\prime}(t)=\bar{U}(t)+\sigma_{U}(t) \tilde{U}^{\prime}(t) \\
& =\bar{U}(t)\left[1+I_{U}(t) \tilde{U}^{\prime}(t)\right]
\end{aligned}
$$

$I_{U}(t)=\sigma_{U}(t) / \bar{U}(t)$ being the slowly-varying turbulent intensity. In this paper, all the slowly-varying quantities are determined through a moving average filter with a moving average period $T=30 \mathrm{~s}$.

The time-varying direction of the velocity $U$ is identified here by the angle $\gamma \in[0,360]$ defined as (Fig. 5):

$\gamma(t)=\operatorname{atan} 2\left[\frac{V_{Y}(t)}{V_{X}(t)}\right]$

where atan 2 is the generalized arc-tangent function.

Figure 6 shows the wind speed classical decomposition of WE1, reporting $V_{X}(\mathrm{a}), V_{Y}(\mathrm{~b}), U(\mathrm{c})$,

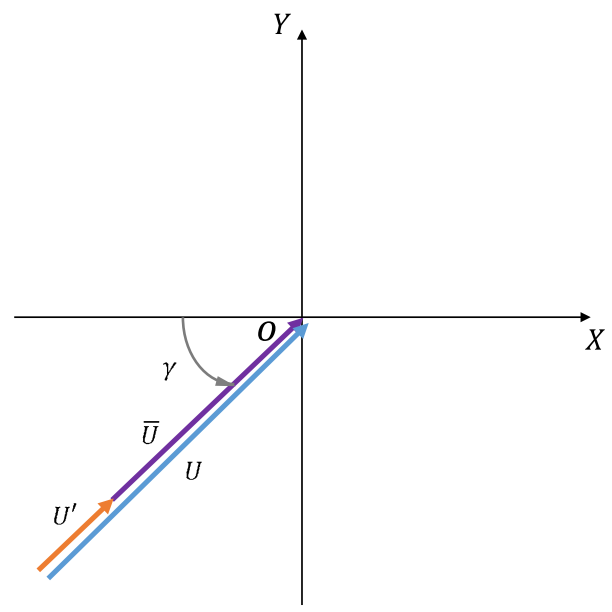

Fig. 5 Classical decomposition method 
Fig. 6 Wind velocity classical decomposition of the record WE1
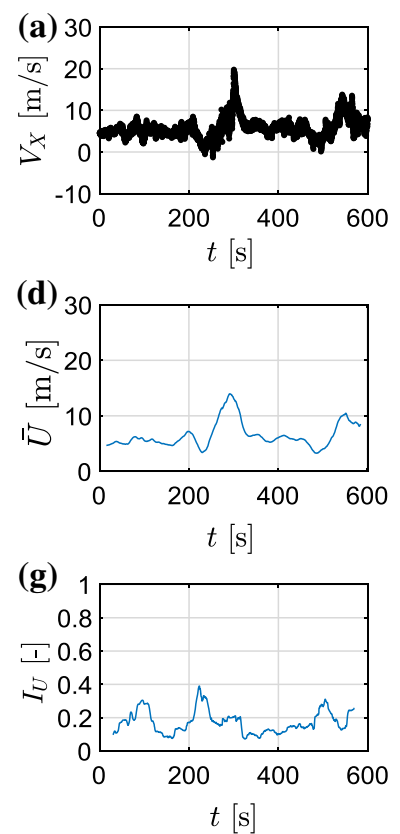

$\bar{U}(\mathrm{~d}), U^{\prime}(\mathrm{e}), \sigma_{U}(\mathrm{f}), I_{U}(\mathrm{~g}), \tilde{U}^{\prime}(\mathrm{h})$ and $\gamma(\mathrm{i})$. The slowlyvarying mean wind velocity has the typical smoothed shape of the instantaneous wind velocity. The maximum value of the slowly-varying mean wind velocity is $\bar{U}_{\max }=13.96 \mathrm{~m} / \mathrm{s}$. The reduced turbulent fluctuation has skewness 0.035 and kurtosis 2.82. The direction diagram shows the strongest gradient during the wind velocity peak phase.

If the directional angle is considered to be constant, the formulation becomes non-directional.

\subsection{Directional decomposition}

This approach [27] consists of decomposing separately the wind speed components $\left(V_{X}, V_{Y}\right)$ into the slowly-varying mean $\left(\bar{V}_{X}, \bar{V}_{Y}\right)$ and residual fluctuation $\left(V_{X}^{\prime}, V_{Y}^{\prime}\right)$ components. The resultant slowly-varying mean wind speed is given by:

$\bar{u}(t)=\sqrt{\bar{V}_{X}^{2}(t)+\bar{V}_{Y}^{2}(t)}$

The slowly-varying direction of $\bar{u}$ is identified by the angle $\bar{\beta} \in[0,360]$ defined as (Fig. 7):
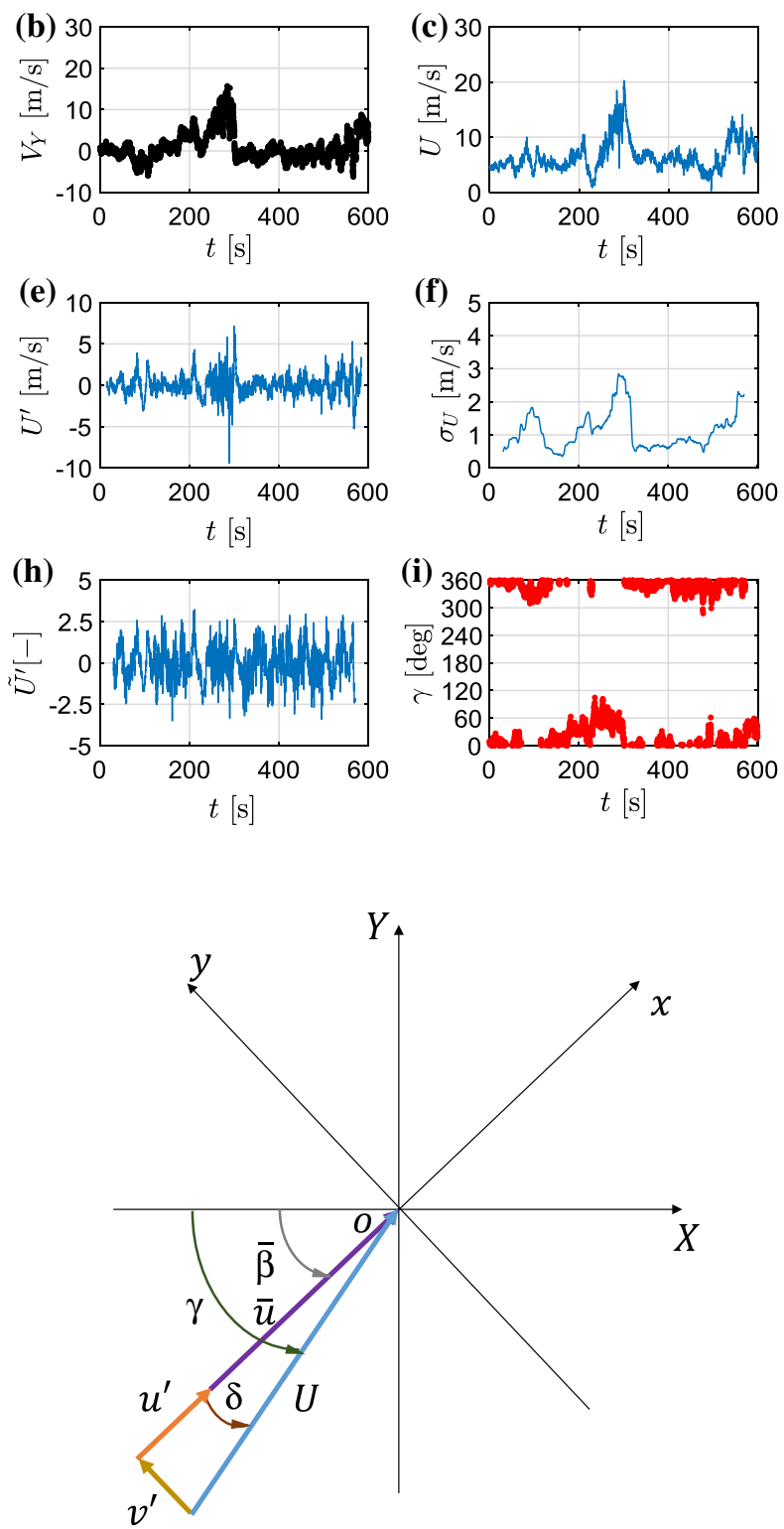

Fig. 7 Directional decomposition method

$\bar{\beta}(t)=\operatorname{atan} 2\left[\frac{\bar{V}_{Y}(t)}{\bar{V}_{X}(t)}\right]$

The residual fluctuation is projected onto a new Cartesian reference system $(x, y)$ where the $x$-axis is aligned with $\bar{u}$. Thus (Fig. 7):

$u^{\prime}(t)=V_{x}^{\prime}(t) \cos \bar{\beta}(t)+V_{y}^{\prime}(t) \sin \bar{\beta}(t)$ 
$v^{\prime}(t)=-V_{x}^{\prime}(t) \sin \bar{\beta}(t)+V_{y}^{\prime}(t) \cos \bar{\beta}(t)$

where $u^{\prime}$ and $v^{\prime}$ are referred to as the longitudinal and lateral turbulence components respectively. They are later expressed as the product of their slowly-varying standard deviations $\left(\sigma_{u}, \sigma_{v}\right)$ by a couple of longitudinal and lateral reduced turbulent fluctuations $\left(\tilde{u}^{\prime}, \tilde{v}^{\prime}\right)$ dealt with as rapidly-varying stationary Gaussian noncorrelated random processes with zero mean and unit standard deviation:

$u^{\prime}(t)=\sigma_{u}(t) \tilde{u}^{\prime}(t)$

$v^{\prime}(t)=\sigma_{v}(t) \tilde{v}^{\prime}(t)$

Accordingly, the longitudinal and lateral components of the wind velocity may be expressed as:

$u(t)=\bar{u}(t)+u^{\prime}(t)=\bar{u}(t)\left[1+I_{u}(t) \tilde{u}^{\prime}(t)\right]$

$v(t)=v^{\prime}(t)=I_{v}(t) \tilde{v}^{\prime}(t)$

where $I_{u}(t)=\sigma_{u}(t) / \bar{u}(t)$ and $I_{v}(t)=\sigma_{v}(t) / \bar{u}(t)$ are respectively the longitudinal and lateral slowly-varying turbulence intensities.

Figure 8 shows the results provided by the directional decomposition of WE1, reporting $\bar{u}(\mathrm{a})$, $u^{\prime}(\mathrm{b}), \sigma_{u}(\mathrm{c}), \bar{\beta}(\mathrm{d}), v^{\prime}(\mathrm{e}), \sigma_{v}(\mathrm{f}), I_{u}(\mathrm{~g}), \tilde{u}^{\prime}(\mathrm{h}), u(\mathrm{i}), \quad I_{v}(\mathrm{j})$, $\tilde{v}^{\prime}(\mathrm{k}), v(\mathrm{l})$. The slowly-varying mean wind velocity follows the same shape as in Fig. 6, but presents a lower maximum value $\bar{u}_{\max }=12.80 \mathrm{~m} / \mathrm{s}$. The reduced longitudinal turbulent fluctuation has skewness 0.007 and kurtosis 2.91, whereas the lateral turbulent fluctuation has skewness -0.0036 and kurtosis 2.81 ; the cross-correlation coefficient of the two reduced turbulent components is -0.067 .

\section{Wind field model}

Section 2.2 provides a description of the 10 records selected amongst the ones collected during the WP [28] and WPS [29] projects, in order to study the directional response to thunderstorm outflows. Starting from these records, this section aims to reconstruct suitable wind fields along the vertical axis of the two structure test cases described in Sect. 2.1. Section 4.1 discusses the use of Monte Carlo simulations by generalizing to directional analysis the non-directional approach introduced in [19, 20]. Section 4.2 overcomes the computational burden of this method, by generalizing to directional analysis the non-directional method introduced in [25] to generate deterministic scenarios that are compatible with single-point records. This approach, applied in Sect. 6, is not so rich as Monte Carlo simulations would have been, but it allows to perform simple evaluations which are well-representative of the structural response [20].

\subsection{Generalized wind field}

In order to provide the aerodynamic loading of slender vertical structures (Sect. 5), the spatial dependence is introduced in Eqs. (2) and (8), obtaining the following expressions:

$$
\begin{aligned}
U(Z, t) & =\bar{U}(Z, t)+U^{\prime}(Z, t) \\
& =\bar{U}(Z, t)\left[1+I_{U}(Z, t) \tilde{U}^{\prime}(Z, t)\right] \\
u(Z, t) & =\bar{u}(Z, t)+u^{\prime}(Z, t) \\
& =\bar{u}(Z, t)\left[1+I_{u}(Z, t) \tilde{u}^{\prime}(Z, t)\right] \\
v(Z, t) & =v^{\prime}(Z, t)=I_{v}(Z, t) \tilde{v}^{\prime}(Z, t)
\end{aligned}
$$

The slowly-varying mean wind velocity is expressed by $[6,18]$ :

$\bar{U}(Z, t)=\bar{U}_{\max }(h) \alpha_{U}(Z) \gamma_{U}(t)$

$\bar{u}(Z, t)=\bar{u}_{\max }(h) \alpha_{u}(Z) \gamma_{u}(t)$

where $\bar{U}_{\text {max }}$ and $\bar{u}_{\text {max }}$ are the maximum values of the slowly-varying mean wind speed, $h$ is the reference height, $\alpha_{U}$ and $\alpha_{u}$ are non-dimensional functions of $Z$ that define the shape of the vertical profile of $\bar{U}$ and $\bar{u}$, $\gamma_{U}$ and $\gamma_{u}$ are non-dimensional time-varying functions, whose maximum value is 1 , which describe how the slowly-varying mean wind speed varies over time.

The functions $\alpha_{U}$ and $\alpha_{u}$ are expressed herein through the model proposed in [34]. It depends on the height $Z$ at which the slowly-varying mean wind speed profile has its maximum (the "height of the nose"). In this paper, this quantity has been assumed to be $25(\mathrm{~m})$. Moreover, it is assumed that the slowly-varying mean wind direction does not change along $Z$ and it is equal to that of the measured record at the reference height $h$.

The slowly-varying turbulence intensity is considered here as independent of height [25] and varying over time according to the relationships:

$I_{U}(Z, t)=\bar{I}_{U}(h) \mu_{U}(t)$ 
Fig. 8 Wind velocity directional decomposition of the record WE1
$I_{u}(Z, t)=\bar{I}_{u}(h) \mu_{u}(t)$

$I_{v}(Z, t)=\bar{I}_{v}(h) \mu_{v}(t)$

where $\bar{I}_{U}, \bar{I}_{u}$ and $\bar{I}_{v}$ are the average values of $I_{U}, I_{u}$ and $I_{v}$ over $\Delta T=10 \mathrm{~min} ; \mu_{U}, \mu_{u}$ and $\mu_{v}$ are non-dimensional functions which express the slow variation of $I_{U}, I_{u}$ and $I_{v}$ over time. Equations (13) and (14) are consistent with field measurements carried out up to a limited height above ground level (nearly $50 \mathrm{~m}$, with a dominant number of anemometers in the first $30 \mathrm{~m}$ ) $[13,35]$. On the other hand, recent CFD simulations through LES [36] and wind tunnel tests using the impinging jet method [37] showed a dependence of the turbulence intensity over height that deserves further studies.

As far as concerns the reduced turbulent fluctuation, there is a wide literature supporting the possibility of expressing the cross-power spectral density (CPSD) of $\tilde{U}^{\prime}$ by the classical models adopted for synoptic winds $[6,13,22,35,38]$. In this paper, this property is generalized to $\tilde{u}^{\prime}$ and $\tilde{v}^{\prime}$.
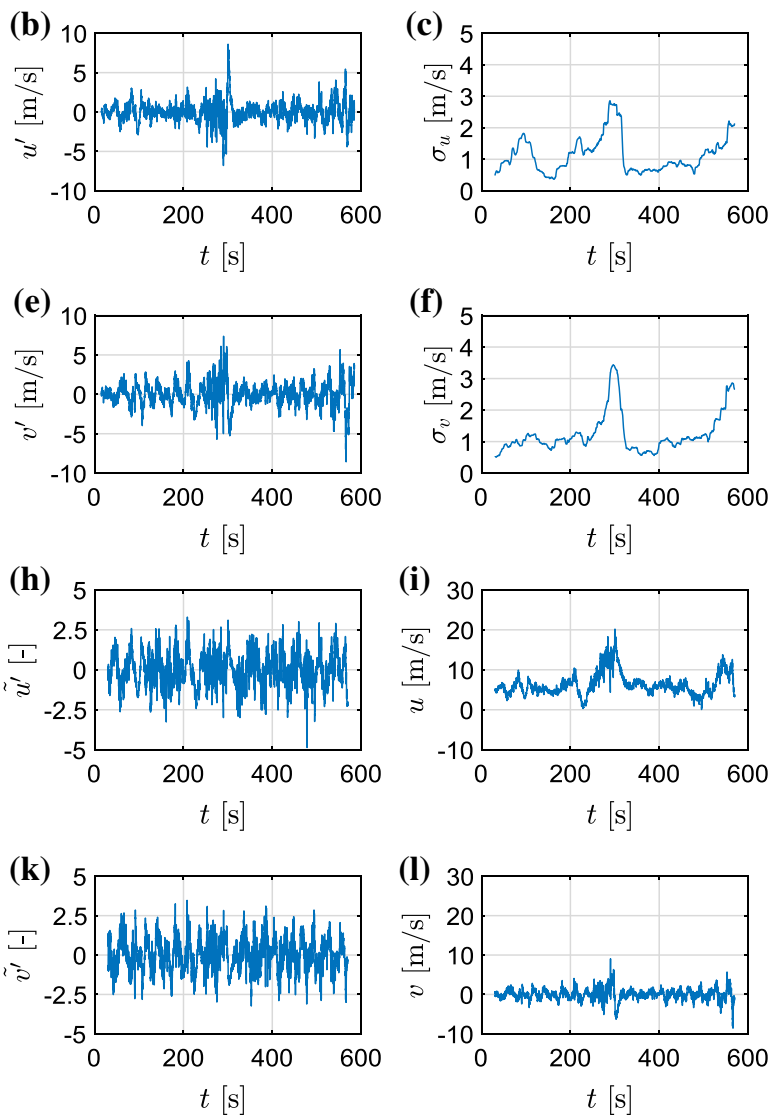

It is worth noting that the classical models adopted in literature to represent the PSD and the coherence function of a synoptic wind speed field involve the presence of a mean wind velocity that, in a stationary flow, is independent of time. During the passage of a gust front, conversely, the (slowly-varying) mean wind velocity varies over time. Thus, the generalization of the spectral properties of a synoptic flow to a thunderstorm outflow involves an approximation according to which, usually, the mean wind velocity is identified with the maximum value of the slowlyvarying mean wind velocity. This approximation, adopted also in the next Sect. 4.2, deserves further investigations. Preliminary evaluations that take into account the transient nature of both the PSD and the coherence function are reported for instance in [39].

\subsection{Equivalent wind field}

To overcome the computational burden of simulating a stationary Gaussian random vector, the equivalent 
wind spectrum technique (EWST) is applied. Introduced in [30] for stationary winds and perfected in [31] for slender structures and structural elements, it replaces the random vector associated to the actual turbulent fluctuation, a random function of time and space, with an equivalent turbulent fluctuation process, identically coherent in space and a random function of time only. This method considers the size of the structure in such a way that its aerodynamic admittance matches the one involved by the actual wind field.

Let us consider the reduced turbulent fluctuation $\tilde{U}^{\prime}$ and let us express its coherence function as:

$\operatorname{Coh}_{\tilde{U}^{\prime} \tilde{U}^{\prime}}\left(Z, Z^{\prime}, n\right)=\exp \left\{-\frac{2 n c_{U z}\left|Z-Z^{\prime}\right|}{\bar{U}_{\max }(Z)+\bar{U}_{\max }\left(Z^{\prime}\right)}\right\}$

$c_{U Z}$ being the exponential decay coefficient of $\tilde{U}^{\prime}$ along $Z$. In this paper $c_{U Z}=10$ [40]. Thus [31]:

$$
\begin{aligned}
U_{e q}(Z, t) & =\bar{U}(Z, t)+U_{e q}^{\prime}(Z, t) \\
& =\bar{U}(Z, t)\left[1+I_{U}(h, t) \tilde{U}_{e q}^{\prime}(t)\right]
\end{aligned}
$$

where $U_{e q}^{\prime}$ is the equivalent turbulent fluctuation and $\tilde{U}_{e q}^{\prime}$ is the equivalent reduced turbulent fluctuation identified by its PSD:

$S_{\tilde{U}_{e q}^{\prime}}\left(n, \delta_{U}\right)=S_{\tilde{U}^{\prime}}(h, n) C\left(\delta_{U} n\right)$

$S_{\tilde{U}^{\prime}}$ is the PSD of $\tilde{U}^{\prime}, C$ is a frequency filter that takes into account the coherence of $\tilde{U}^{\prime}$ and reduces its PSD in equivalent terms:

$C(\eta)=\frac{1}{\eta}-\frac{1}{2 \eta^{2}}\left(1-e^{-2 \eta}\right)(\eta>0) ; C(0)=1$

$\delta_{U}$ is the size factor:

$\delta_{U}=\frac{\kappa c_{U Z} H}{\bar{U}_{\max }\left(Z_{e q}\right)}$

$\kappa$ is a coefficient associated with the first modal shape:

$\kappa=\frac{0.5}{\left(\zeta_{1}+1\right)^{0.55}}$

where $\zeta_{1}$ is a parameter defined in Sect. 2.1. $Z_{e q}=$ $0.6 H$ is the equivalent height.

Figure 9a shows the actual reduced turbulent fluctuation extracted from the record WE1 whereas
Fig. 9b, c shows the equivalent reduced turbulent fluctuation respectively for $\mathrm{S} 1$ and $\mathrm{S} 2$.

Generalizing this formulation to the longitudinal and lateral reduced turbulence components, $\tilde{u}^{\prime}$ and $\tilde{v}^{\prime}$, Eq. (8) may be re-written as:

$$
\begin{aligned}
u_{e q}(Z, t) & =\bar{u}(Z, t)+u_{e q}^{\prime}(Z, t) \\
& =\bar{u}(Z, t)\left[1+I_{u}(h, t) \tilde{u}_{e q}^{\prime}(t)\right] \\
v_{e q}(Z, t) & =v_{e q}^{\prime}(Z, t)=\bar{u}(Z, t) I_{v}(h, t) \tilde{v}_{e q}^{\prime}(t)
\end{aligned}
$$

where $u_{e q}^{\prime}$ and $v_{e q}^{\prime}$ are the longitudinal and lateral equivalent turbulent fluctuations; $\tilde{u}_{e q}^{\prime}$ and $\tilde{v}_{e q}^{\prime}$ are the longitudinal and lateral equivalent reduced turbulent fluctuations identified by their PSD:

$S_{\tilde{u}_{e q}^{\prime}}\left(n, \delta_{u}\right)=S_{\tilde{u}^{\prime}}(h, n) C\left(\delta_{u} n\right)$

$S_{\tilde{v}_{e q}^{\prime}}\left(n, \delta_{v}\right)=S_{\tilde{v}^{\prime}}(h, n) C\left(\delta_{v} n\right)$

$S_{\tilde{u}^{\prime}}$ and $S_{\tilde{v}^{\prime}}$ are the PSD of $\tilde{u}^{\prime}$ and $\tilde{v}^{\prime}, \delta_{u}$ and $\delta_{v}$ are the size factors defined as:

$\delta_{u}=\frac{\kappa c_{u Z} H}{\bar{u}_{\max }\left(Z_{e q}\right)}$

$\delta_{v}=\frac{\kappa c_{v Z} H}{\bar{u}_{\max }\left(Z_{e q}\right)}$

$c_{u Z}$ and $c_{v Z}$ are the exponential decay coefficients, respectively, of $\tilde{u}^{\prime}$ and $\tilde{v}^{\prime}$ along $Z$. In this paper $c_{u Z}=10$ and $c_{v Z}=6.5$ [40].

The explicit representation of $\tilde{U}_{e q}^{\prime}, \tilde{u}_{e q}^{\prime}$ and $\tilde{v}_{e q}^{\prime}$ may be carried out through the classical Monte Carlo simulation of stationary Gaussian random processes [19], or by the deterministic representation introduced in [25]. Following the latter approach $\tilde{U}_{e q}^{\prime}, \tilde{u}_{e q}^{\prime}$ and $\tilde{v}_{e q}^{\prime}$ are expressed as the inverse Fourier transforms of, respectively, $\hat{\tilde{U}}_{e q}^{\prime}, \hat{\tilde{u}}_{e q}^{\prime}$ and $\hat{\tilde{v}}_{e q}^{\prime}$, which are evaluated as:

$\hat{\tilde{U}}_{e q}^{\prime}\left(n, \delta_{U}\right)=\hat{\tilde{U}}^{\prime}(h, n) \sqrt{C\left(\delta_{U} n\right)}$

$\hat{\tilde{u}}_{e q}^{\prime}\left(n, \delta_{u}\right)=\hat{\tilde{u}}^{\prime}(h, n) \sqrt{C\left(\delta_{u} n\right)}$

$\hat{\tilde{v}}_{e q}^{\prime}\left(n, \delta_{v}\right)=\hat{\tilde{v}}^{\prime}(h, n) \sqrt{C\left(\delta_{v} n\right)}$

where $\hat{\tilde{U}^{\prime}}$, $\hat{\tilde{u}}^{\prime}$ and $\hat{\tilde{v}}^{\prime}$ are the Fourier transforms, respectively, of the actual reduced turbulent fluctuations $\tilde{U}^{\prime}, \tilde{u}^{\prime}$ and $\tilde{v}^{\prime}$. 
Fig. 9 Actual reduced turbulent fluctuation a and equivalent reduced turbulent fluctuation for b S1 and $\mathbf{c ~ S 2}$
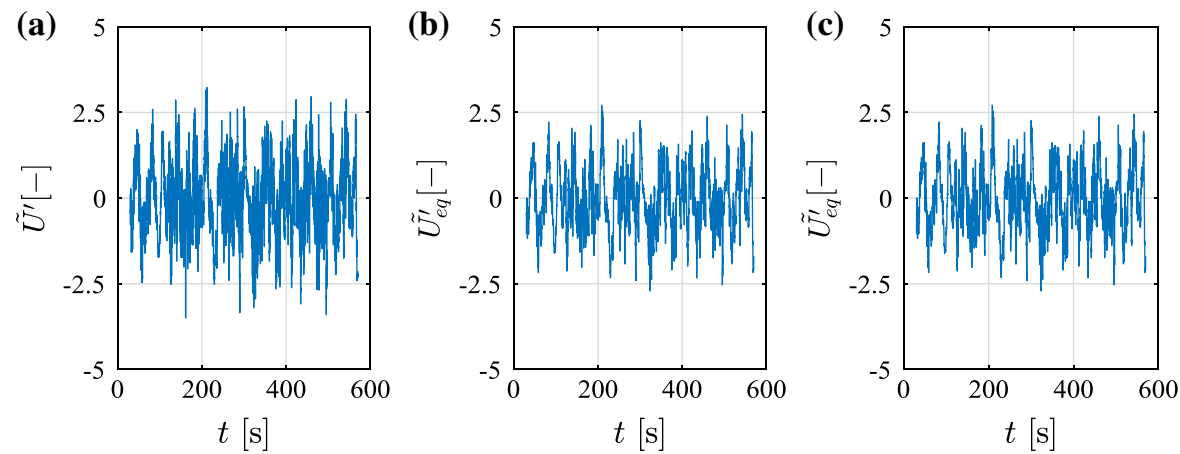

\section{Aerodynamic wind loading}

The object of this section is to develop an appropriate aerodynamic loading assessment for each different wind field model introduced in Sect. 3 and to compare their properties.

Let us consider a slender vertical structure whose cross-section $\mathrm{C}$ has the reference dimension $b$ and the centre of gravity in $O$. The $Z$-axis identifies the vertical extension of the structure and passes through $O$. The position of $O$ does not change along $Z$. The structure is subjected to a force $F$ and to a torsional moment $M$ per unit length along $Z$ that are applied in the plane of the cross-section $\mathrm{C}$. The torsional moment $M$ is here disregarded due to the compactness and torsional stiffness of the cross-section whereas the force $F$ may be projected on three different couples of Cartesian axes (Fig. 10). The first reference system is identified by the fixed $X$ - and $Y$-axes along the principal directions of the cross-section (Fig. 4); let $f_{X}$ and $f_{Y}$ be the force per unit length along $X$ and $Y$. In the second reference system the $x$-axis is aligned with the slowly-varying mean wind speed $\bar{u}$ whereas the $y$-axis is perpendicular to it; the angle of attack $\bar{\beta}$ defines the orientation of $\bar{u}$ with respect to $X$; let $f_{x}$ and $f_{y}$ be the alongwind and crosswind forces per unit length along $x$ and $y$. In the third reference system the $d$-axis is aligned with the instantaneous resultant wind speed $U$ whereas the $l$-axis is perpendicular to it; the angle of attack $\gamma$ defines the orientation of $U$ with respect $X$; let $f_{d}$ and $f_{l}$ be the drag and lift forces per unit length along $d$ and $l$. Let $\delta=\gamma-\bar{\beta}$ be the angle between the $x$ - and $d$-axes. It is worth mentioning that the $X$ - and $Y$-axes are fixed and so they do not change over time; the $x$ and $y$-axes, relevant only for the directional

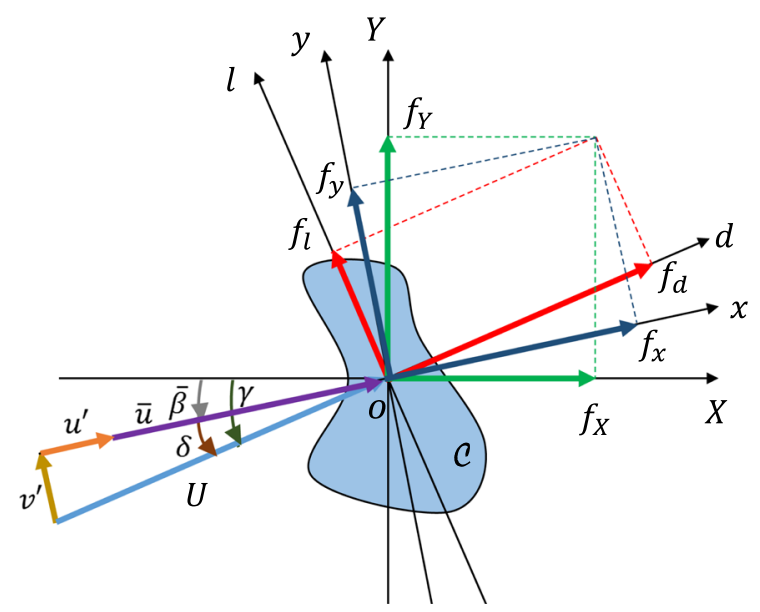

Fig. 10 Reference system for the cross-section of a slender vertical structure

decomposition, slowly change their direction over time; the $d$ - and $l$-axes rapidly change their direction over time.

In the following sub-sections, the aerodynamic wind loading associated with the different wind field models described in Sects. 3 and 4 are evaluated through strip and quasi-steady theory [41]. Vortex shedding effects [3] are neglected. Considering also the properties of the structures dealt with in Sect. 2.1, the occurrence of the Reynolds' crisis phenomenon [42] is excluded for sake of simplification. The effects of transient aerodynamics [22] are considered by invoking the hypothesis that the passage of the gust front is moderately slow [13, 35, 43]; accordingly, the aerodynamic coefficients are expressed here as slowly-varying functions of time. This is made possible by assuming that the Reynolds number is associated with the slowly-varying mean wind 
velocity [44] whereas the angle of attack is associated with the slowly-varying mean wind direction [27].

In the following sub-sections the aerodynamic loading is first expressed with reference to the generalized wind field model described in Sect. 4.1, than simplified by making recourse to the EWST (Sect. 4.2).

\subsection{Classical non-directional method (Method 0)}

Let us consider the classical decomposition (Sect. 3.1). If the angle of attack variation is disregarded, the dynamic response implicitly occurs in the alongwind direction. Very often, this latter is assumed to be the same of one of the principal axes of the structure cross-section.

Let us doing this assumption and consider the angle of attack $\gamma=0$. The time-varying drag and lift forces per unit length result:

$f_{d}(Z, t)=\frac{1}{2} \rho b(Z) U^{2}(Z, t) c_{d}(Z, t)$

$f_{l}(Z, t)=\frac{1}{2} \rho b(Z) U^{2}(Z, t) c_{l}(Z, t)$

where $\rho$ is the density of air, $U$ is the wind velocity defined by Eq. (9), $c_{d}$ and $c_{l}$ are evaluated in correspondence of a Reynolds number associated to $\bar{U}$, Eq. (11), and to $\gamma=0$, Eq. (3).

Because of the hypotheses previously mentioned, the aerodynamic wind loading along $X$ coincides with the drag force and the aerodynamic wind loading along $Y$ coincides with the lift force (Fig. 11):

$f_{X}(Z, t)=f_{d}(Z, t)$

$f_{Y}(Z, t)=f_{l}(Z, t)$

It is worth noting that classical formulations neglect the aerodynamic lift force independently of the shape of the structure cross-section [2]. Actually, this position is correct provided that $X$ is a (geometrical) symmetry axis. In the present case this situation happens for both the considered structure test cases. So the lift force is rigorously nil.

The application of the EWST is implemented by replacing $U$ in Eq. (26) with $U_{e q}$, Eq. (16).

\subsection{Classical method including wind direction} (Method 1)

Including the presence of the wind direction within the classical framework, the time-varying angle of attack $\gamma$ is given by Eq. (3) (Fig. 5). Thus, the drag and lift forces per unit length are provided by Eq. (26), where $c_{d}$ and $c_{l}$ are evaluated in correspondence of a Reynolds number associated to $\bar{U}$, Eq. (11), and to $\bar{\gamma}$ extracted from $\gamma$, Eq. (3), through a moving average filter.

The projection of the drag and lift forces onto the principal directions provides (Fig. 12):

$f_{X}(Z, t)=f_{d}(Z, t) \cos [\gamma(Z, t)]-f_{l}(Z, t) \sin [\gamma(Z, t)]$

$f_{Y}(Z, t)=f_{d}(Z, t) \sin [\gamma(Z, t)]+f_{l}(Z, t) \cos [\gamma(Z, t)]$

where, conventionally, $\gamma(Z, t)=\gamma(h, t)$. It is worth noting that the aerodynamic coefficients are evaluated with regard to $\bar{\gamma}$ whereas the drag and lift forces are projected onto the principal axes by using $\gamma$.

\subsection{Directional method (method 2)}

Let us consider the directional decomposition method described in Sect. 3.2. Its outcome is a resultant velocity which is composed of a slowly varying mean part and a residual turbulent fluctuation. The latter is not collinear with the slowly-varying mean wind speed, but it is made up of a longitudinal and a lateral component, $u^{\prime}$ and $v^{\prime}$. The direction of the slowlyvarying mean wind speed with respect to $X$ is identified by the slowly-varying angle of attack $\bar{\beta}$. The resultant velocity is oriented, respect to $X$, with an angle of attack $\gamma$ which does not coincide with $\bar{\beta}$ because of the presence of $v^{\prime}$ (Fig. 7). The angle $\delta$ is the difference between $\gamma$ and $\bar{\beta}$ and is defined as:

$\delta(Z, t)=\tan ^{-1}\left[\frac{v^{\prime}(Z, t)}{\bar{u}(Z, t)+u^{\prime}(Z, t)}\right]$

The drag and lift forces per unit length are expressed by Eq. (26), where $c_{d}$ and $c_{l}$, diversely from Sect. 5.2, are evaluated in correspondence of a Reynolds number associated to $\bar{u}$, Eq. (12), and to $\bar{\beta}$, Eq. (5). Considering that $\bar{u} \approx \bar{U}$ and $\bar{\beta} \approx \bar{\gamma}$ especially when turbulence is small (Sect. 5.4), it seems quite 
(a)

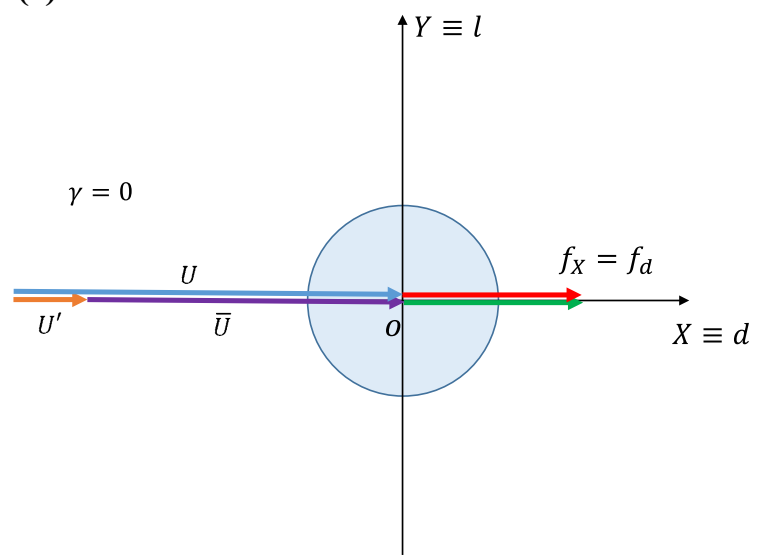

(b)

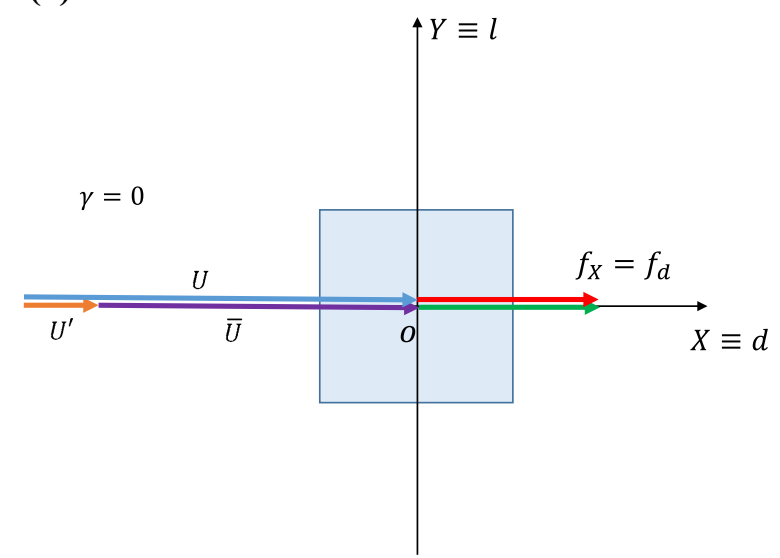

Fig. 11 Classical decomposition for a a circular and $\mathbf{b}$ squared cross-section
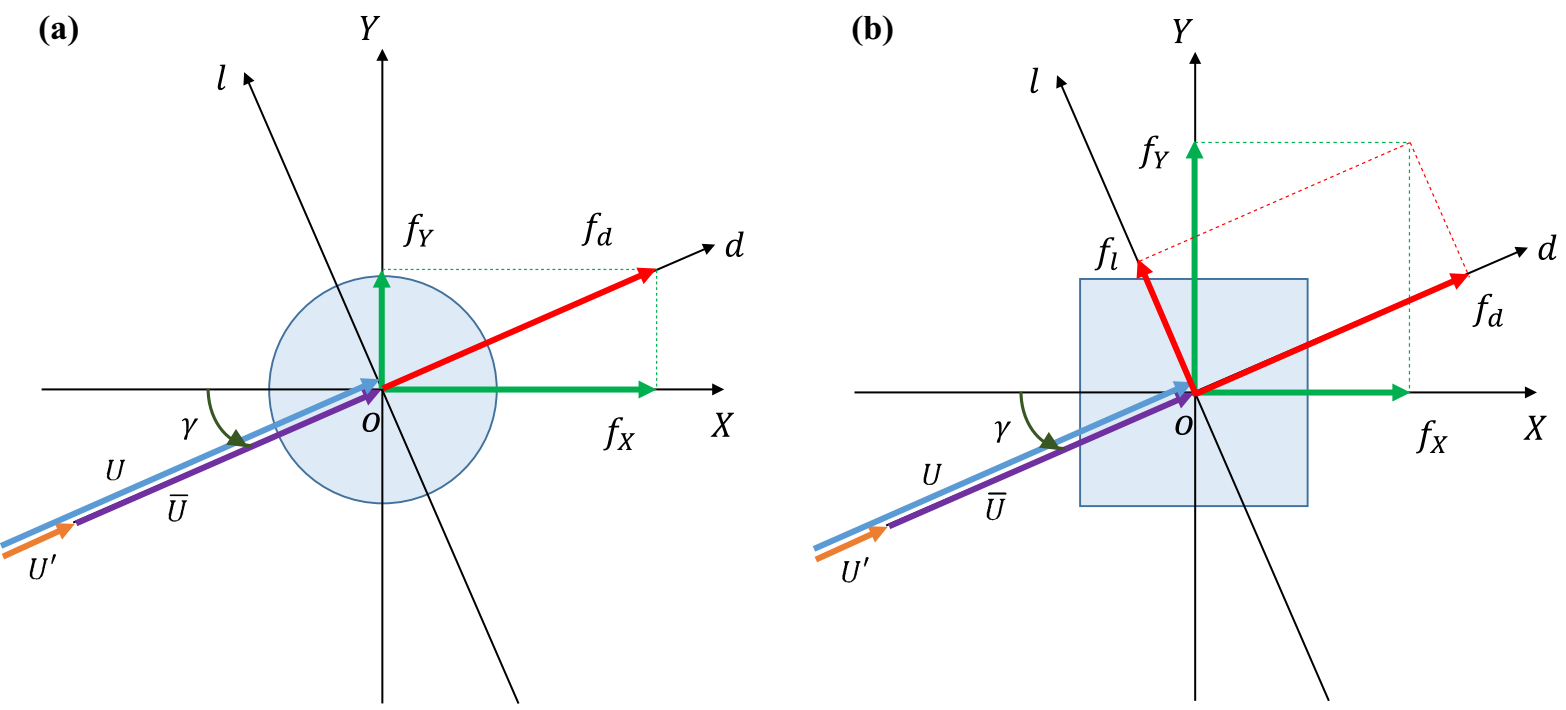

Fig. 12 Classical decomposition applied on a $\mathbf{a}$ circular and $\mathbf{b}$ squared cross-section

reasonable to expect that Methods 1 and 2 provide similar results.

The projection of the drag and lift forces onto $x$ and $y$ provides the alongwind and crosswind forces:

$f_{x}(Z, t)=f_{d}(Z, t) \cos [\delta(Z, t)]-f_{l}(Z, t) \sin [\delta(Z, t)]$

$f_{y}(Z, t)=f_{l}(Z, t) \cos [\delta(Z, t)]+f_{d}(Z, t) \sin [\delta(Z, t)]$

$f_{x}(Z, t)=\frac{1}{2} \rho b(Z) U^{2}(Z, t) c_{x}(Z, t)$

$f_{y}(Z, t)=\frac{1}{2} \rho b(Z) U^{2}(Z, t) c_{y}(Z, t)$

where $c_{x}$ and $c_{y}$ are the force coefficients in the $x$ - and $y$-directions:

$c_{x}(Z, t)=c_{d}(Z, t) \cos [\delta(Z, t)]-c_{l}(Z, t) \sin [\delta(Z, t)]$

Alternatively, Eq. (30) may be expressed as: 
$c_{y}(Z, t)=c_{l}(Z, t) \sin [\delta(Z, t)]+c_{d}(Z, t) \cos [\delta(Z, t)]$

Independently of using Eq. (30) or Eq. (31), the forces along $X$ and $Y$ result (Fig. 13):

$f_{X}(Z, t)=f_{x}(Z, t) \cos [\bar{\beta}(t)]-f_{y}(Z, t) \sin [\bar{\beta}(t)]$

$f_{Y}(Z, t)=f_{y}(Z, t) \cos [\bar{\beta}(t)]+f_{x}(Z, t) \sin [\bar{\beta}(t)]$

The application of the EWST is easily implemented by replacing $U$ in Eq. (31) with $U_{e q}$, now provided by the resultant of $u_{e q}$ and $v_{e q}$, Eq. (21), and $u^{\prime}$ and $v^{\prime}$ in Eq. (29) with $u_{e q}^{\prime}$ and $v_{e q}^{\prime}$, Eq. (21). It is worth noting that in this case the angle $\delta$ in Eq. (29) depends on the sole $t$ :

$$
\delta_{e q}(t)=\tan ^{-1}\left[\frac{I_{v}(h, t) \tilde{v}_{e q}^{\prime}(t)}{1+I_{u}(h, t) \tilde{u}_{e q}^{\prime}(t)}\right] .
$$

\subsection{Directional method under the small turbulence hypothesis (Method 3)}

The small turbulence hypothesis is typically made in the study of synoptic events because it leads to simplified analyses - the loading retains the Gaussian PDF that is typical of the wind speed-and limited

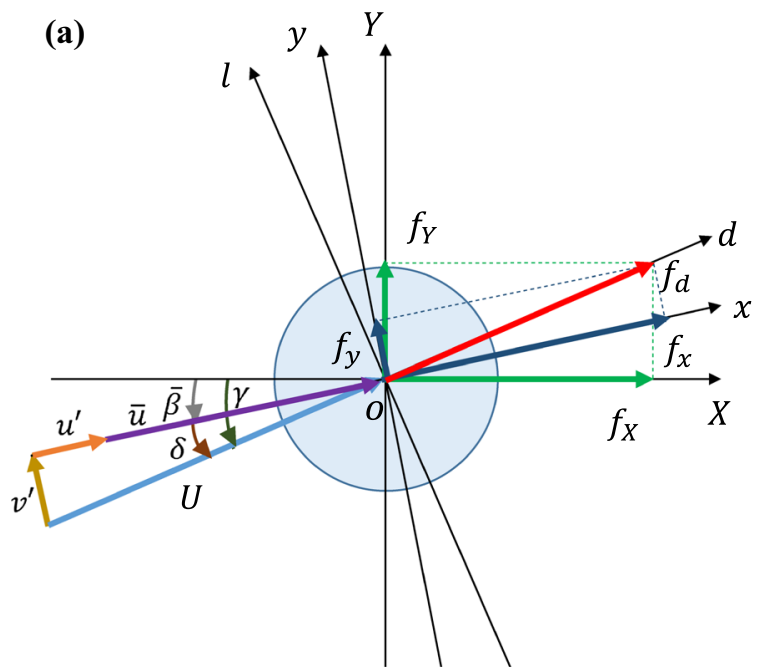

errors in the evaluation of both the aerodynamic loading and the dynamic response [45, 46].

In order to apply the above hypothesis in the present generalized framework, let us expand the force coefficients defined by Eq. (32) via Taylor series around $\gamma=\bar{\beta}$ and the $\delta$ angle defined by Eq. (29) via McLaurin series around $\delta=0$; besides, let us express the wind speed in Eq. (31) as:

$U(Z, t)=\sqrt{\left[\bar{u}(Z, t)+u^{\prime}(Z, t)\right]^{2}+v^{\prime 2}(Z, t)}$

Finally, let us introduce the small turbulence hypothesis assuming that:

$\frac{u^{\prime}(Z, t)}{\bar{u}(Z, t)} \ll 1$

$\frac{v^{\prime}(Z, t)}{\bar{u}(Z, t)} \ll 1$

Accordingly, Eq. (31) becomes:

$$
\begin{aligned}
f_{x}(Z, t)= & \frac{1}{2} \rho b(Z) \bar{u}^{2}(Z, t) c_{d}(Z, t) \\
+ & \rho b(Z) \bar{u}(Z, t) u^{\prime}(Z, t) c_{d}(Z, t) \\
& +\frac{1}{2} \rho b(Z) \bar{u}(Z, t) v^{\prime}(Z, t)\left[c_{d}^{\prime}(Z, t)-c_{l}(Z, t)\right]
\end{aligned}
$$

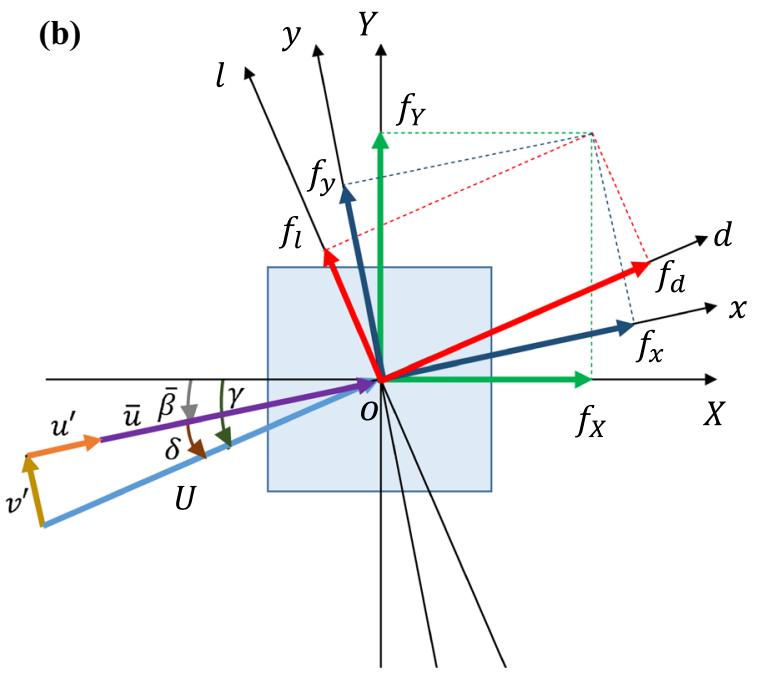

Fig. 13 Directional decomposition applied on $\mathbf{a}$ a circular and $\mathbf{b}$ squared cross-section 


$$
\begin{aligned}
f_{y}(Z, t)= & \frac{1}{2} \rho b(Z) \bar{u}^{2}(Z, t) c_{l}(Z, t) \\
& +\rho b(Z) \bar{u}(Z, t) u^{\prime}(Z, t) c_{l}(Z, t) \\
& +\frac{1}{2} \rho b(Z) \bar{u}(Z, t) v^{\prime}(Z, t)\left[c_{d}(Z, t)+c_{l}^{\prime}(Z, t)\right]
\end{aligned}
$$

Equation (37) constitutes the generalization of the classical formulas of the alongwind and crosswind forces traditionally applied for synoptic winds. In this latter case such forces correspond to the sum of three terms: the first expresses the mean static loading, the second the fluctuating part of the stationary loading proportional to the longitudinal turbulence $u^{\prime}$, the third the fluctuating part of the stationary loading proportional to the lateral turbulence $v^{\prime}$.

In the case of thunderstorm outflows the nonstationarity character of the wind velocity makes the first term a slowly-varying function of time of quasistatic nature. The second and the third term are nonstationary processes proportional to the (non-stationary) longitudinal turbulence $u^{\prime}$ and to the (nonstationary) lateral turbulence $v^{\prime}$, respectively. In addition, due to the slowly-varying mean wind direction $\bar{\beta}$, the alongwind and the crosswind force slowly vary their direction over time.

This latter condition has a relevant consequence. In the case of synoptic winds the problem is drastically simplified provided that the mean wind velocity is aligned with a cross-section symmetry axis; in such case $c_{l}=c_{d}^{\prime}=0$. In the case of thunderstorm outflows this simplification cannot occur due to the time evolution of $\bar{\beta}$.

As in Sect. 5.3, Eq. (33) provides the forces along the principal $X$ - and $Y$-axes (Fig. 13).

The application of the EWST is implemented by replacing $u^{\prime}$ and $v^{\prime}$ in Eq. (37) with $u_{e q}^{\prime}$ and $v_{e q}^{\prime}$, Eq. (21). This leads to re-write Eq. (37) as:

$$
\begin{aligned}
& f_{x}(Z, t)=\frac{1}{2} \rho b(Z) \bar{u}^{2}(Z, t) . \\
& \left\{c_{d}(Z, t)+2 I_{u}(h, t) \tilde{u}_{e q}^{\prime}(t) c_{d}(Z, t)\right. \\
& \left.+I_{v}(h, t) \tilde{v}_{e q}^{\prime}(t)\left[c_{d}^{\prime}(Z, t)-c_{l}(Z, t)\right]\right\}
\end{aligned}
$$

$$
\begin{aligned}
& f_{y}(Z, t)=\frac{1}{2} \rho b(Z) \bar{u}^{2}(Z, t) . \\
& \left\{c_{l}(Z, t)+2 I_{u}(h, t) \tilde{u}_{e q}^{\prime}(t) c_{l}(Z, t)\right. \\
& \left.+I_{v}(h, t) \tilde{v}_{e q}^{\prime}(t)\left[c_{d}(Z, t)+c_{l}^{\prime}(Z, t)\right]\right\}
\end{aligned}
$$

Accordingly, the application of the EWST highlights a property already implicit in Eq. (37): the non-stationary loading terms proportional to the longitudinal and to the lateral turbulence components are in reality stationary processes, $\tilde{u}_{e q}^{\prime}$ and $\tilde{v}_{e q}^{\prime}$, modulated by slowly-varying functions of time.

A dominant property of Method 3 is that, diversely from the previous methods whose assessment implies more or less conventional and even arbitrary assumptions, it involves the sole small turbulence hypothesis.

\section{Dynamic response}

This section studies the dynamic response of a slender vertical structure, in particular S1 and S2 (Sect. 2.1), subjected to the aerodynamic loading of thunderstorm outflows, in particular WE1-W10 (Sect. 2.2). Section 6.1 describes the method applied to perform the dynamic analysis and its qualitative results with regard to WE1. Section 6.2 gathers the results obtained for all the 10 signals investigated providing quantitative comparisons and examinations.

\subsection{Time-domain integration of the equations of motion}

Let us consider a slender vertical structure with linear elastic behaviour. In each $X, Z$ and $Y, Z$ plane the natural frequencies are well-separated. The damping is small and proportional. Being $X$ and $Y$ principal axes and neglecting aeroelastic effects (e.g. the aerodynamic damping), the $X$ - and $Y$-components of the displacement at quote $Z$ are uncoupled of each other and are given by:

$q_{i}(Z, t)=\sum_{k} \psi_{k, i}(Z) p_{k, i}(t)$

where $\psi_{k, i}$ is the $k$-th mode shape in the $i=X, Y$ direction and $p_{k, i}$ is the $k$-th principal coordinate in the $i$ direction. The latter term is furnished by the solution of the following equation: 

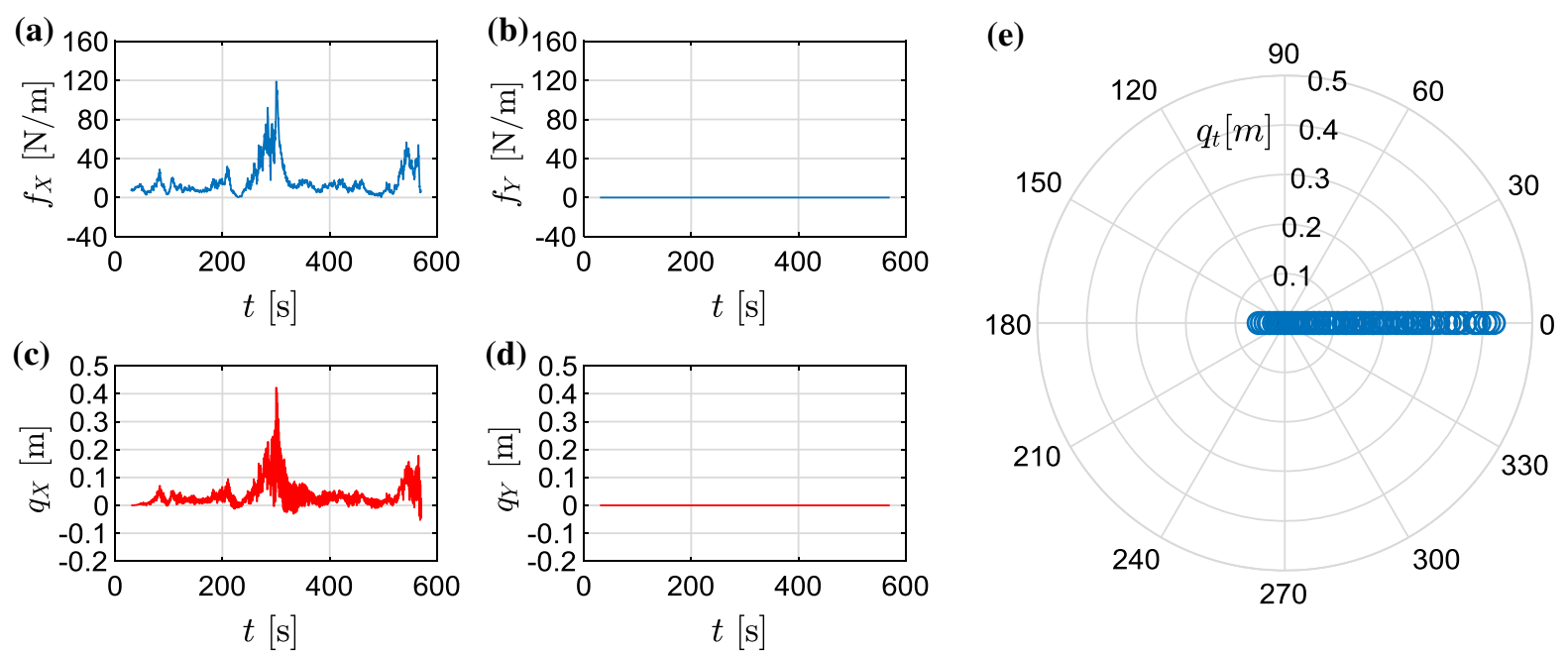

Fig. 14 Dynamic response of S1 to WE1 by Method 0: a force per unit length at the structure top along $X$-axis and b $Y$-axis; c, d corresponding displacements; e polar plot of the response

$\ddot{p}_{k, i}(t)+2 \xi_{k, i} \omega_{k, i} \dot{p}_{k, i}(t)+\omega_{k, i}^{2} p_{k, i}(t)=\frac{1}{m_{k, i}} f_{k, i}(t)$

where $m_{k, i}$ is $k$-th modal mass in the $i$ direction and $f_{k, i}$ is the $k$-th modal force in the $i$ direction:

$m_{k, i}=\int_{0}^{H} m(Z) \psi_{k, i}^{2}(Z) d Z$

$f_{k, i}(t)=\int_{0}^{H} f_{i}(Z, t) \psi_{k, i}(Z) d Z$

$m$ is the mass per unit length, $f_{X}$ and $f_{Y}$ are the aerodynamic wind forces per unit length defined in Sect. 5 for the different studied methods.

The resultant displacement is determined by combining its $X$ - and $Y$-components as:

$q_{t}(Z, t)=\sqrt{q_{X}^{2}(Z, t)+q_{Y}^{2}(Z, t)}$

Coherently with [19, 20, 25] the following analyses consider the sole contribution of the first mode of vibration in each principal direction.

The dynamic response is evaluated in the timedomain, through the integration of the equations of motion in the state space with a time step $\Delta t=0.1 \mathrm{~s}$. In order to avoid transient effects in the initial phase of the response, a cosine function window has been introduced at the beginning of each wind loading history.
For sake of comparison and homogeneity, the ten thunderstorm outflow signals examined here (Sect. 2.2) have been scaled to the same conventional peak wind velocity equal to $40(\mathrm{~m} / \mathrm{s})$.

Figures 14, 15, 16, 17, 18, 19, 20 and 21 show the dynamic response of the test structures $\mathrm{S} 1$ and $\mathrm{S} 2$ with regard to WE1 - for each of the methods described in Sect. 5-by reporting: the non-windowed time-histories of $f_{X}$ and $f_{Y}$ at the structure top (a, b), the timehistory of $q_{X}$ and $q_{Y}$ at the structure top (c, d); a polar plot of the top displacement that enhances its direction pattern (e) ( $X$ left to right, $Y$ bottom to top).

A comprehensive overview of Figs. 14, 15, 16, 17, $18,19,20$ and 21 points out three different levels of remarks.

First of all, the differences between the results provided by the non-directional Method 0 and the directional Methods 1, 2 and 3 are apparent. Figures 14 and 15, referred to Method 0, show that the loading and the response occur, for both $\mathrm{S} 1$ and $\mathrm{S} 2$, in the sole alongwind direction $X$. Figures $16,17,18,19$, 20 and 21, referred to Methods 1 to 3, show loading and displacement components in both the $X$ and $Y$ directions. The comparison between the polar diagrams (e) in Figs. 14 and 15 and in Figs. 16, 17, $18,19,20$ and 21 confirms this remark in an expressive way.

The second aspect that emerges from this overview is the different behaviour of S1 (Figs. 14, 16, 18 and 20) and S2 (Figs. 15, 17, 19 and 21). Since S1 is a 

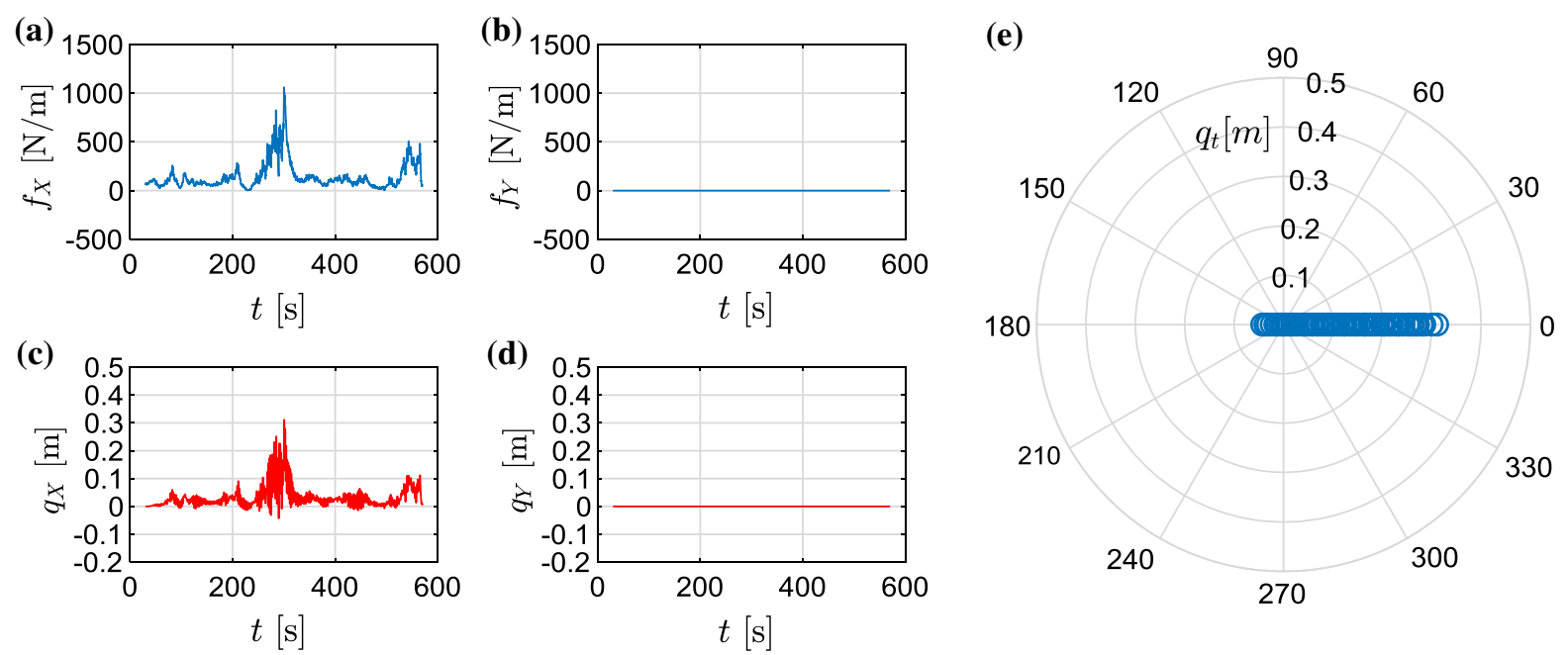

Fig. 15 Dynamic response of S2 to WE1 by Method 0: a force per unit length at the structure top along $X$-axis and b $Y$-axis; c, d corresponding displacements; e polar plot of the response
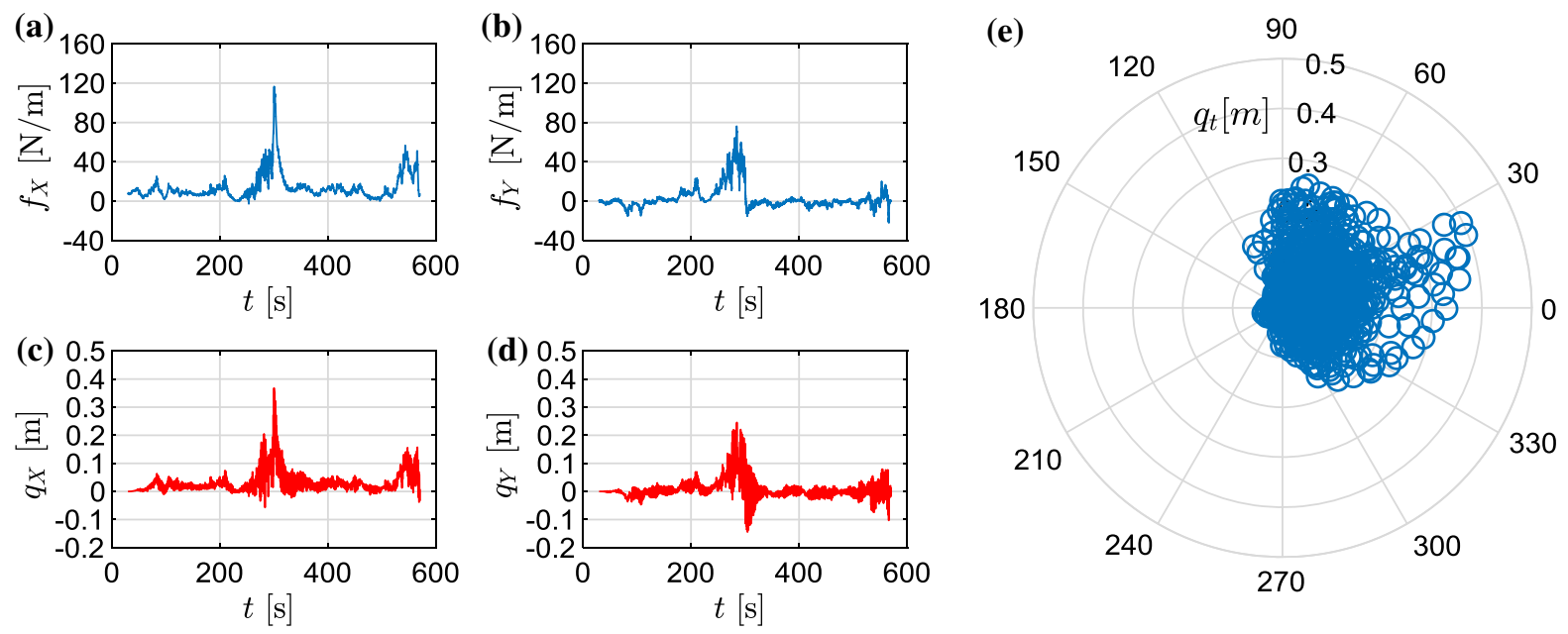

Fig. 16 Dynamic response of S1 to WE1 by Method 1: a force per unit length at the structure top along $X$-axis and b $Y$-axis; c, d corresponding displacements; e polar plot of the response

polar-symmetric plan structure, the role of directionality effects is much less pronounced than for $\mathrm{S} 2$. Accordingly, Methods 1 to 3 provide results substantially comparable with those obtained through Method 0 . This does not apply to $\mathrm{S} 2$, for which the discrepancies between the two different philosophies emerge. This happens not only due to the direction changes of the analysed event, but because its angle of attack involves, for more or less long periods, directional sectors more or less favourable from the aerodynamic viewpoint. For instance, when the wind speed attacks S2 along its main diagonal $\left(\gamma=45^{\circ}\right)$ the alongwind loading is greater than in the case in which the wind speed is perpendicular to a structure face; the latter in turn represents the persistent situation evaluated by Method 0. Also in this case the comparison between the polar diagrams (e) for S1 and S2 confirms this remark in an expressive way.

The third aspect that deserves a remark is the comparison between different directional methods (Figs. 16, 17, 18, 19, 20 and 21). It is worth noticing that each one of them, being based on more or less different wind and loading models, captures forces and displacements in a different way for each structure. All 

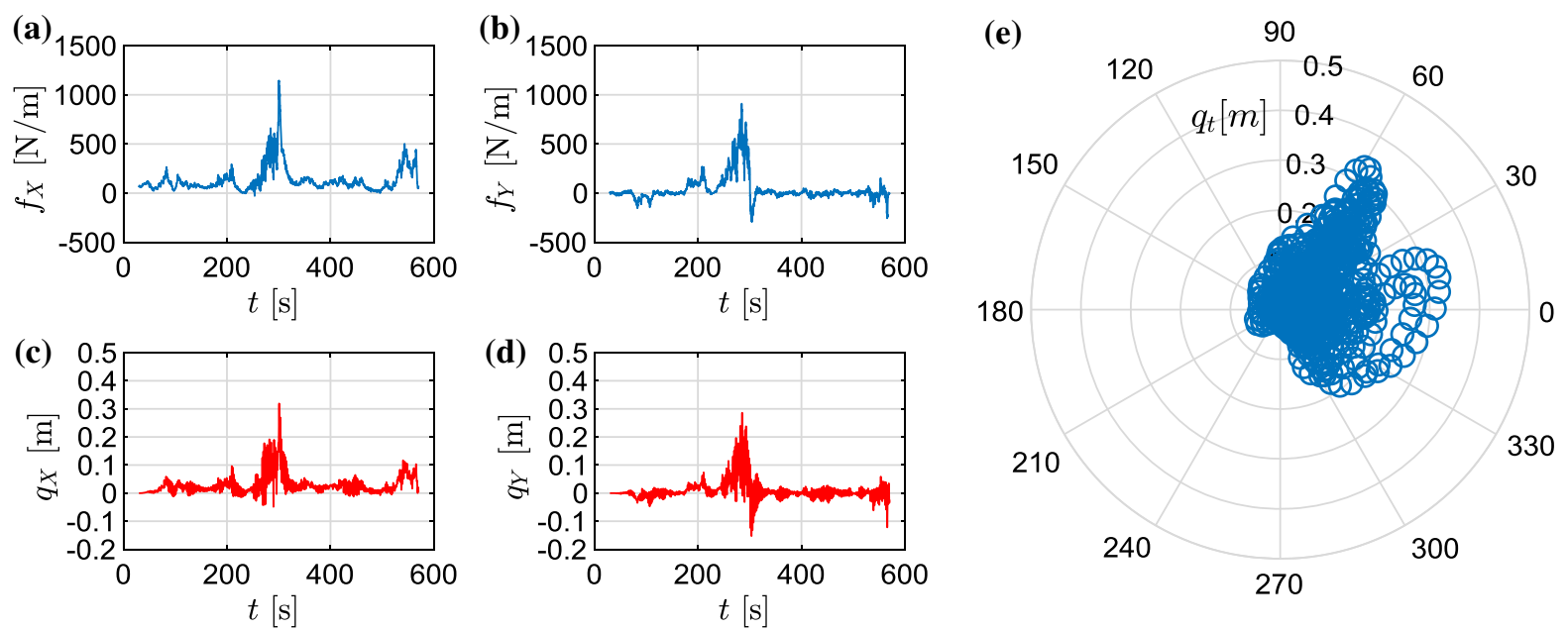

Fig. 17 Dynamic response of S2 to WE1 by Method 1: a force per unit length at the structure top along $X$-axis and b $Y$-axis; c, d corresponding displacements; e polar plot of the response
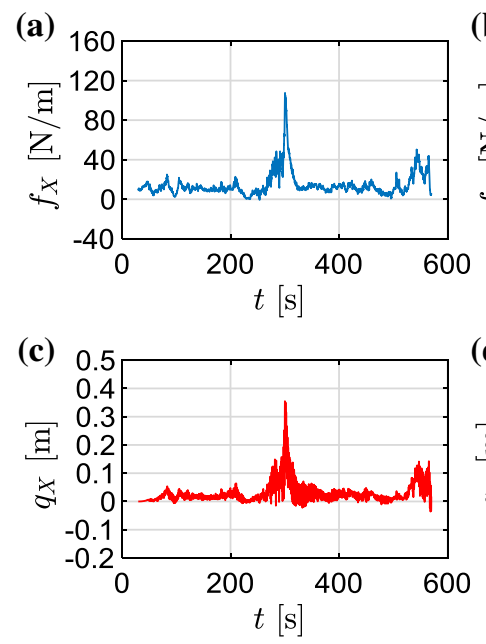
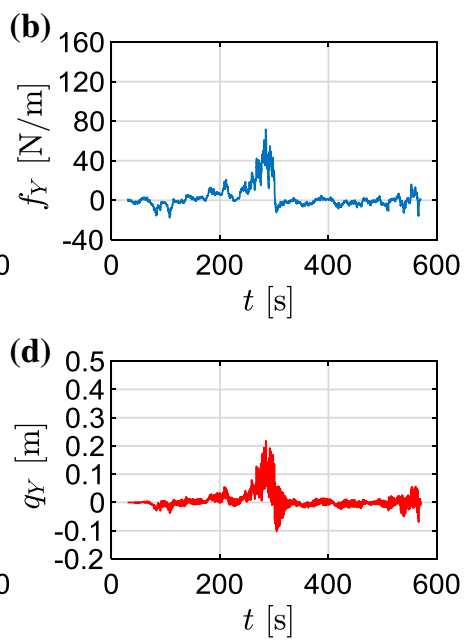

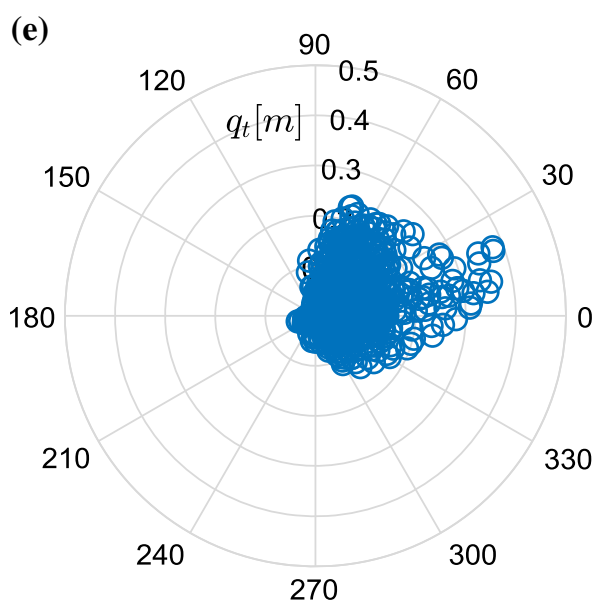

Fig. 18 Dynamic response of S1 to WE1 by Method 2: a force per unit length at the structure top along $X$-axis and b $Y$-axis; c, d corresponding displacements; e polar plot of the response

together they exhibit a common average behaviour but different quantitative values. This situation is clearly influenced by the properties peculiar of WE1. This calls for interpreting the spread of the results with reference to several thunderstorm outflows. This matter is dealt with in the next Sect. 6.2.

\subsection{Overall results and discussion}

Tables 3 and 4 show, respectively for S1 and S2, the maximum resultant displacement $q_{t}(\mathrm{~m})$ at the structure top, evaluated for the different methods described in Sect. 5, for the entire set of the 10 thunderstorm outflows. Next to $q_{t}$, between brackets, the percentage difference between the results obtained by Methods 1 to 3 and by Method 0 is reported. The last two rows show, for each method, the mean value and the coefficient of variation (cov) of the resultant displacement and, between brackets, the percentage difference referred to Method 0.

The mean values of the resultant displacement in Tables 3 and 4 confirm and quantify, firstly, the diverse behaviour of S1 and S2. In the polar-symmetric plan structure (S1) the various proposed methods 

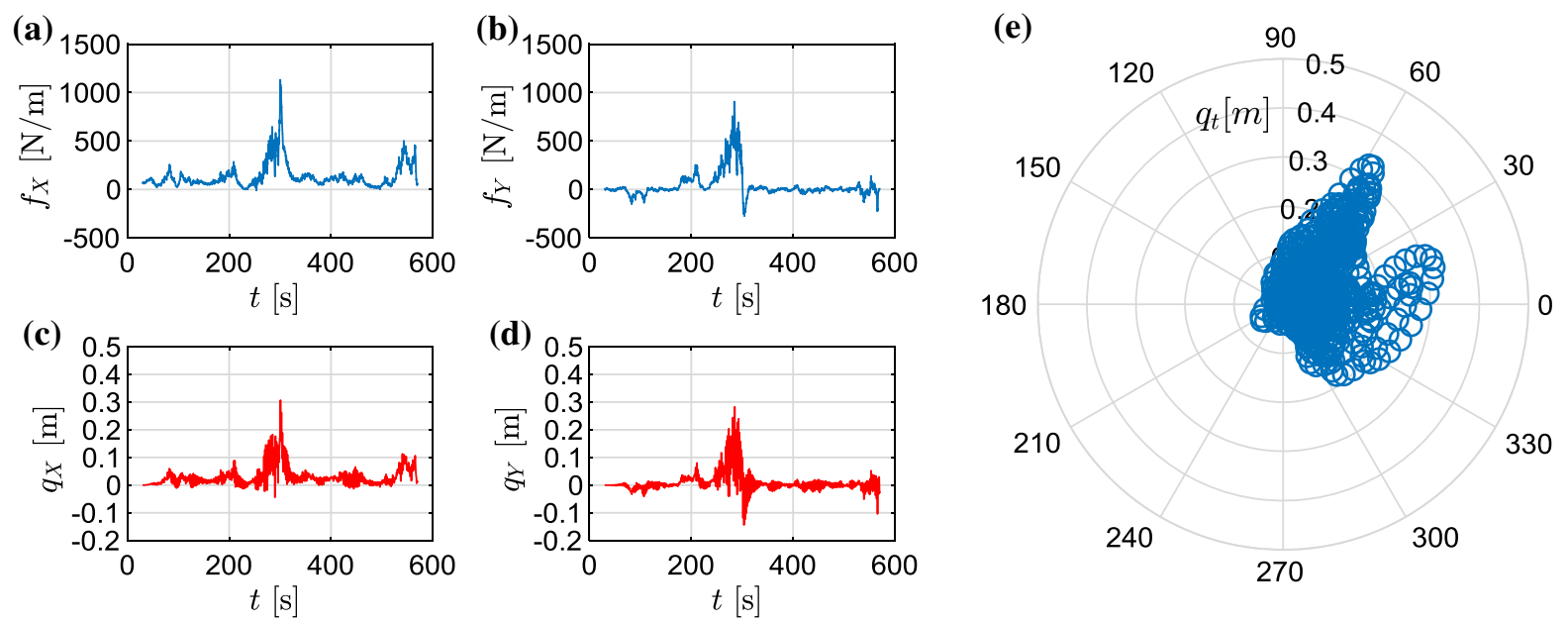

Fig. 19 Dynamic response of S2 to WE1 by Method 2: a force per unit length at the structure top along $X$-axis and b $Y$-axis; c, d corresponding displacements; e polar plot of the response
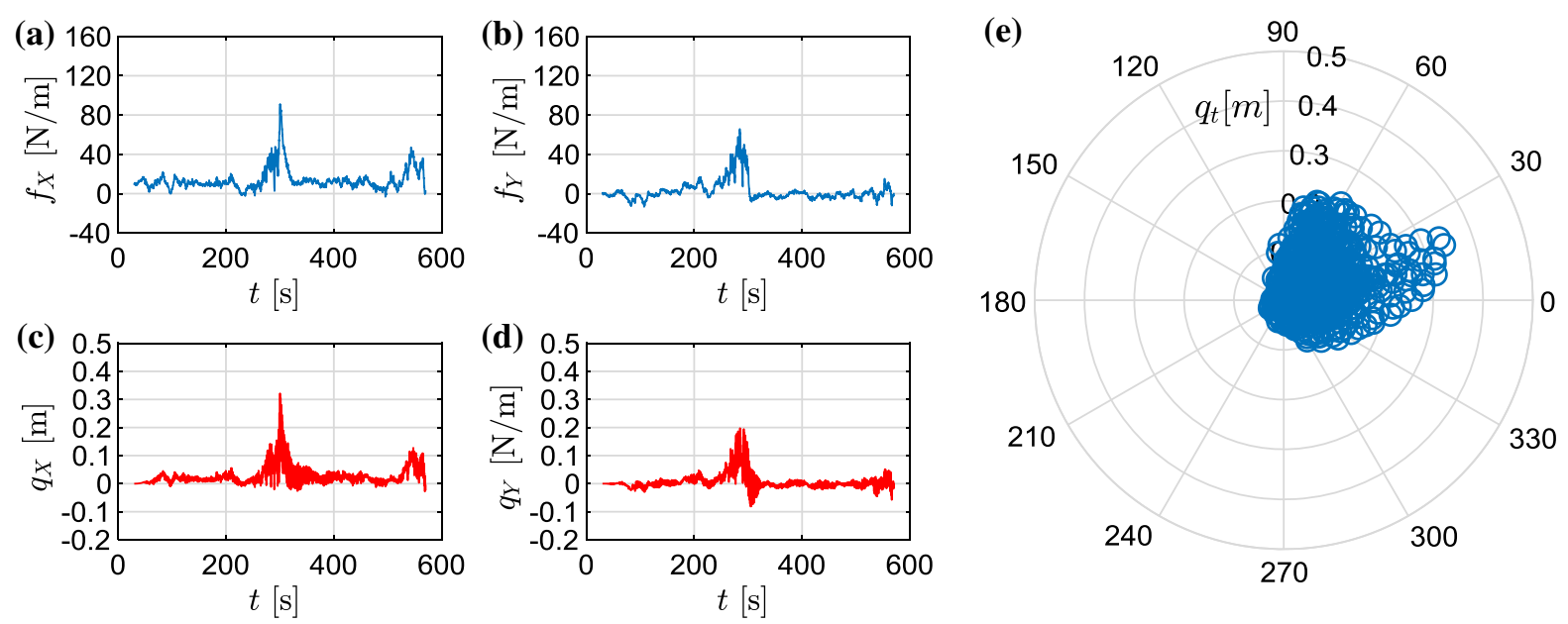

Fig. 20 Dynamic response of $\mathrm{S} 1$ to WE1 by Method 3: a force per unit length at the structure top along $X$-axis and b $Y$-axis; c, d corresponding displacements; e polar plot of the response

lead to similar results; in no case differences between directional and non-directional analyses exceed $4.2 \%$. In the square-section plan structure (S2) the role of the wind direction is instead relevant. All the proposed directional methods (Methods 1 to 3 ) lead to significantly higher displacement values than those evaluated in non-directional form (Method 0).

Focusing on S2, Methods 1 to 3 exceed the results of Method 0, respectively, on average by $17.1 \%$, $16.4 \%$ and $13.1 \%$. As expected, Methods 1 and 2 provide very similar results. Method 3, which differs from Method 2 because it considers the turbulence as small entails a $3 \%$ average reduction with respect to Method 2. In this regard it is interesting to point out that such reduction is reasonable and expected, but does not seem to be so obvious. In particular, considering WE4 and WE9, treating the turbulence as small reduces the aerodynamic loading but slightly increases the response. This effect may be interpreted observing that removing the quadratic term of the turbulence the harmonic content of the loading is also modified: this is not relevant for static and quasi-static behaviours but may be not negligible for dynamic evaluations. Another potential interpretation may be 

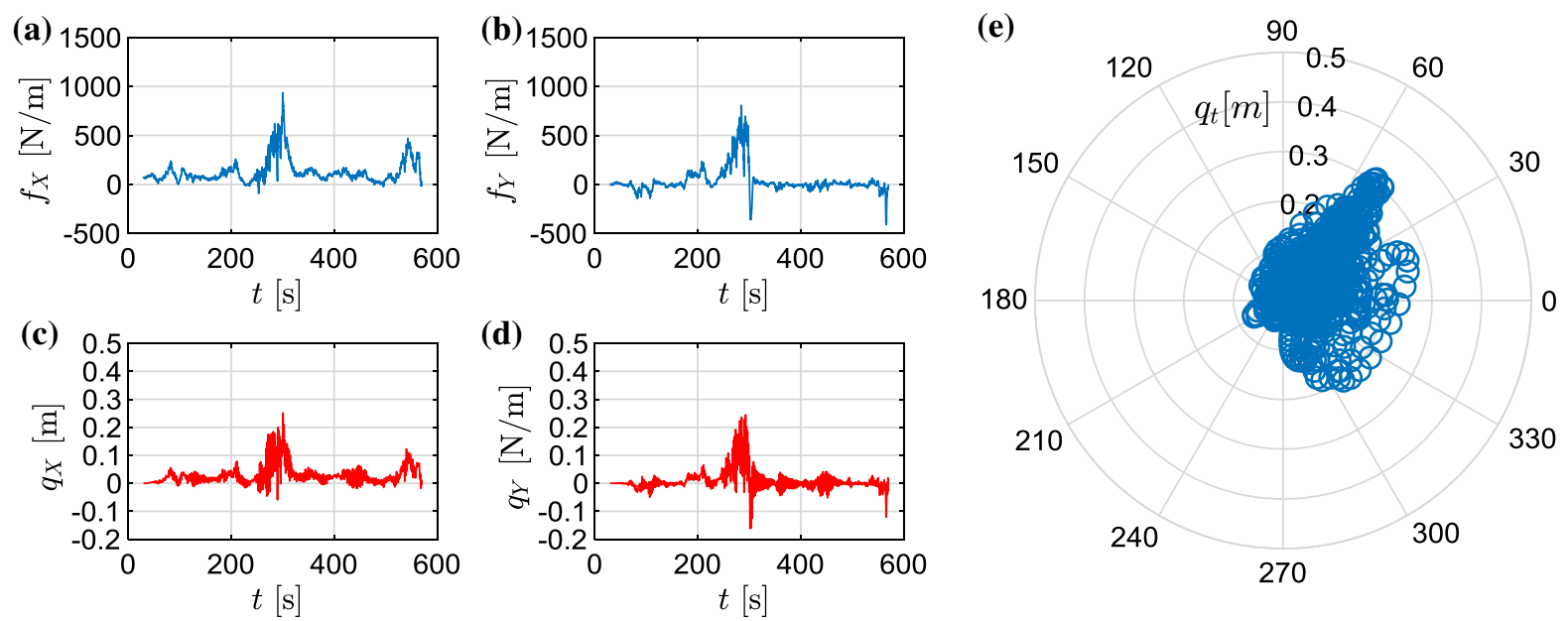

Fig. 21 Dynamic response of S2 to WE1 by Method 3: a force per unit length at the structure top along $X$-axis and b $Y$-axis; c, d corresponding displacements; e polar plot of the response

Table 3 Maximum displacement (m) (cov in the last row) at S1 top and percentage difference between Methods 1, 2, 3 and Method 0

Table 4 Maximum displacement $(\mathrm{m})$ (cov in the last row) at S2 top and percentage difference between Methods 1, 2, 3 and Method 0

\begin{tabular}{lllll}
\hline Wind event & Method 0 & Method 1 & Method 2 & Method 3 \\
\hline WE1 & 0.41 & $0.38(-6.1 \%)$ & $0.38(-6.9 \%)$ & $0.34(-16.4 \%)$ \\
WE2 & 0.34 & $0.35(0.3 \%)$ & $0.35(0.5 \%)$ & $0.34(-0.5 \%)$ \\
WE3 & 0.33 & $0.34(3.4 \%)$ & $0.34(1.6 \%)$ & $0.33(0.8 \%)$ \\
WE4 & 0.33 & $0.33(1.1 \%)$ & $0.33(0.8 \%)$ & $0.32(-3.6 \%)$ \\
WE5 & 0.35 & $0.36(3.1 \%)$ & $0.35(0.7 \%)$ & $0.35(-1.2 \%)$ \\
WE6 & 0.38 & $0.39(3.7 \%)$ & $0.38(0.9 \%)$ & $0.34(-11.9 \%)$ \\
WE7 & 0.32 & $0.34(5.8 \%)$ & $0.32(-0.3 \%)$ & $0.31(-2.9 \%)$ \\
WE8 & 0.39 & $0.40(1.4 \%)$ & $0.40(0.9 \%)$ & $0.40(2.0 \%)$ \\
WE9 & 0.31 & $0.31(1.3 \%)$ & $0.31(0.8 \%)$ & $0.31(-0.6 \%)$ \\
WE10 & 0.28 & $0.35(23.2 \%)$ & $0.29(4.1 \%)$ & $0.27(-4.9 \%)$ \\
Mean & 0.34 & $0.35(3.2 \%)$ & $0.34(0.1 \%)$ & $0.33(-4.2 \%)$ \\
Cov & 0.109 & $0.076(-30.3 \%)$ & $0.094(-13.8 \%)$ & $0.099(-9.2 \%)$ \\
\hline
\end{tabular}

\begin{tabular}{lllll}
\hline Wind event & Method 0 & Method 1 & Method 2 & Method 3 \\
\hline WE1 & 0.31 & $0.33(7.2 \%)$ & $0.33(7.5 \%)$ & $0.31(-0.9 \%)$ \\
WE2 & 0.31 & $0.31(-1.1 \%)$ & $0.31(0.0 \%)$ & $0.30(-2.1 \%)$ \\
WE3 & 0.32 & $0.32(0.0 \%)$ & $0.32(-1.5 \%)$ & $0.31(-5.3 \%)$ \\
WE4 & 0.30 & $0.36(19.9 \%)$ & $0.36(19.0 \%)$ & $0.38(24.8 \%)$ \\
WE5 & 0.29 & $0.38(32.2 \%)$ & $0.38(32.3 \%)$ & $0.38(33.9 \%)$ \\
WE6 & 0.30 & $0.33(8.0 \%)$ & $0.33(7.6 \%)$ & $0.33(8.7 \%)$ \\
WE7 & 0.32 & $0.38(19.9 \%)$ & $0.38(21.0 \%)$ & $0.37(16.2 \%)$ \\
WE8 & 0.32 & $0.42(33.4 \%)$ & $0.42(32.0 \%)$ & $0.41(30.7 \%)$ \\
WE9 & 0.30 & $0.38(26.0 \%)$ & $0.38(25.2 \%)$ & $0.40(30.6 \%)$ \\
WE10 & 0.34 & $0.43(26.4 \%)$ & $0.42(22.3 \%)$ & $0.33(-1.7 \%)$ \\
Mean & 0.31 & $0.36(17.1 \%)$ & $0.36(16.4 \%)$ & $0.35(13.1 \%)$ \\
Cov & 0.043 & $0.108(151 \%)$ & $0.101(135 \%)$ & $0.109(153 \%)$ \\
\hline
\end{tabular}


related to the fact that neglecting the quadratic term of the turbulence the wind direction is also modified; in this regard it is worth noting that the polar symmetric structure $\mathrm{S} 1$ does not exhibit any situation in which the reduction of the loading due to assuming the turbulence as small leads to increasing the response. Many other numerical tests, not reported here, have been carried out for synoptic winds and thunderstorm outflows, using directional and non-directional decomposition rules. All of them confirmed the presence of singular limited cases in which assuming the turbulence as small leads to increase the response. Although all these cases involve very small changes, in the order of a few percent, this matter deserves more investigations.

Inspired by this remark and moving on to the values of the displacement in relation to single events, their dispersion, quantified by the cov values in Tables 3 and 4 , is apparent from event to event and from method to method. For instance, focusing on S2, Method 3 exceeds the results of Method 0 by over $30 \%$ for WE5, WE8 and WE9 whereas it reduces its results for WE2 and WE3. This seems particularly significant, recalling that the velocities of all the events have been normalized to the same peak velocity value. This confirms and possibly enhances one of the most relevant results discussed in $[19,20]$ : the spread of the maximum value of the response to thunderstorm outflows is much greater than that for synoptic winds $[47,48]$. Other analyses not reported here show that normalising the wind velocities of all the events to the same maximum value of the slowly-varying mean wind velocity makes the spread of the maximum value of the displacement nearly double than the one provided by Tables 3 and 4; this result confirms previous outcomes discussed in [20].

In the light of the analyses carried out and of the results obtained with regard to 10 thunderstorm scenarios the changes in direction during the passage of the gust front cannot be ignored (if not for structures with polar-symmetric cross-section) and must be properly evaluated in terms of wind speed, aerodynamic loading and dynamic response. Quantitatively more robust results in respect of the errors committed neglecting directional effects call for extensive Monte Carlo simulations $[19,20]$ aiming to interpret this issue in a probabilistic framework.

\section{Conclusions and perspectives}

This paper generalizes the classic assessment of the stationary wind-excited response of structures to synoptic winds, conceived without taking the change of the wind direction into account, to the nonstationary wind-excited response of structures to thunderstorm outflows, where the change of the wind direction due to combining the background wind, the translation of the downburst and the radial nature of the downdraft after the touchdown is a peculiar property that structures frequently experience during the passage of the gust front.

This study identifies two different philosophiesthe former based on the classical approach to thunderstorm outflows and the latter innovative-that are later on traced back to four methods. Method 0 neglects the wind directionality and deals with the response as implicitly alongwind. Method 1 includes the wind directionality into Method 0 without changing its traditional spirit. Method 2 implements a novel decomposition strategy of the wind speed that leads to express the aerodynamic loading in terms of alongwind and crosswind forces, the direction of which slowly varies over the time. Method 3 introduces the classical small turbulence hypothesis into Method 2, establishing a robust parallelism with the classical analysis of synoptic winds.

Numerical analyses are carried out with regard to two slender vertical structures-one endowed with a circular cross-section (S1) and the other with a square cross-section (S2) - that are subjected to 10 thunderstorm outflow scenarios.

The role of the wind directionality is limited for $\mathrm{S} 1$, but is pronounced for S2. In this latter case the directional Methods 1 to 3 provide results substantially different from those obtained by means of the non-directional Method 0. Focusing on the average behaviour of S2, Methods 1 to 3 exceed the results of Method 0 respectively by $17.1,16.4$ and $13.1 \%$. Methods 1 and 2 provide similar results. Method 3 reduces the results of Method 2 by $3 \%$. Well beyond these average estimates, the dispersion of the results is apparent and very wide when considering the single thunderstorm scenarios. This confirms and possibly enhances the property according to which the spread of the maximum value of the response to thunderstorm outflows is much greater than that for synoptic winds. 
Summarising the whole of these results directionality effects due to the passage of the gust front cannot be ignored (if not for structures with polar-symmetric cross-section) and must be properly evaluated in terms of wind speed, aerodynamic loading and dynamic response. More robust and precise results in respect of the errors committed neglecting the directional effects cannot avoid extensive Monte Carlo simulations aiming to interpret this issue in a probabilistic framework.

This remark identifies the first and main perspective that emerges from this research with regard to its prosecution: the transition from deterministic scenarios to probabilistic simulations. This does not exclude many other issues that remain open and seem to be of considerable importance. Among these, the study of the variation of the wind direction with height during the passage of the gust front and the role of the aeroelastic phenomena neglected herein stand out.

Acknowledgements This research is funded by European Research Council under the European Union's Horizon 2020 research and innovation program (Grant Agreement No. 741273) for the project THUNDERR - Detection, simulation, modelling and loading of thunderstorm outflows to design wind safer and cost-efficient structures - through an Advanced Grant 2016. It is co-funded by the Italian Ministry of Instruction and Scientific Research (MIUR), Prot. 2015TTJN95 in the framework of the Research Project of Relevant National
Interest (PRIN 2015) "Identification and Diagnostic of Complex Structural Systems", The data used for this research were recorded by the monitoring network set up as part of the European Projects Winds and Ports (Grant No. B87E09000000007) and Wind, Ports and Sea (Grant No. B82F13000100005), funded by the European Territorial Cooperation Objective, Cross-border program Italy-France Maritime 2007-2013.

\section{Compliance with ethical standards}

Conflict of interest The authors declare that they have no conflict of interest.

Open Access This article is distributed under the terms of the Creative Commons Attribution 4.0 International License (http:// creativecommons.org/licenses/by/4.0/), which permits unrestricted use, distribution, and reproduction in any medium, provided you give appropriate credit to the original author(s) and the source, provide a link to the Creative Commons license, and indicate if changes were made.

\section{Appendix: Selected wind velocity records}

Figure 22 shows the resultant wind speed (left column) and direction (right column) of the ten thunderstorm outflows selected (Sect. 2.2) for the present analyses. 
Fig. 22 Resultant wind speed (left column) and direction (right column) of WE1 (a), WE2 (b),...WE10 (j)
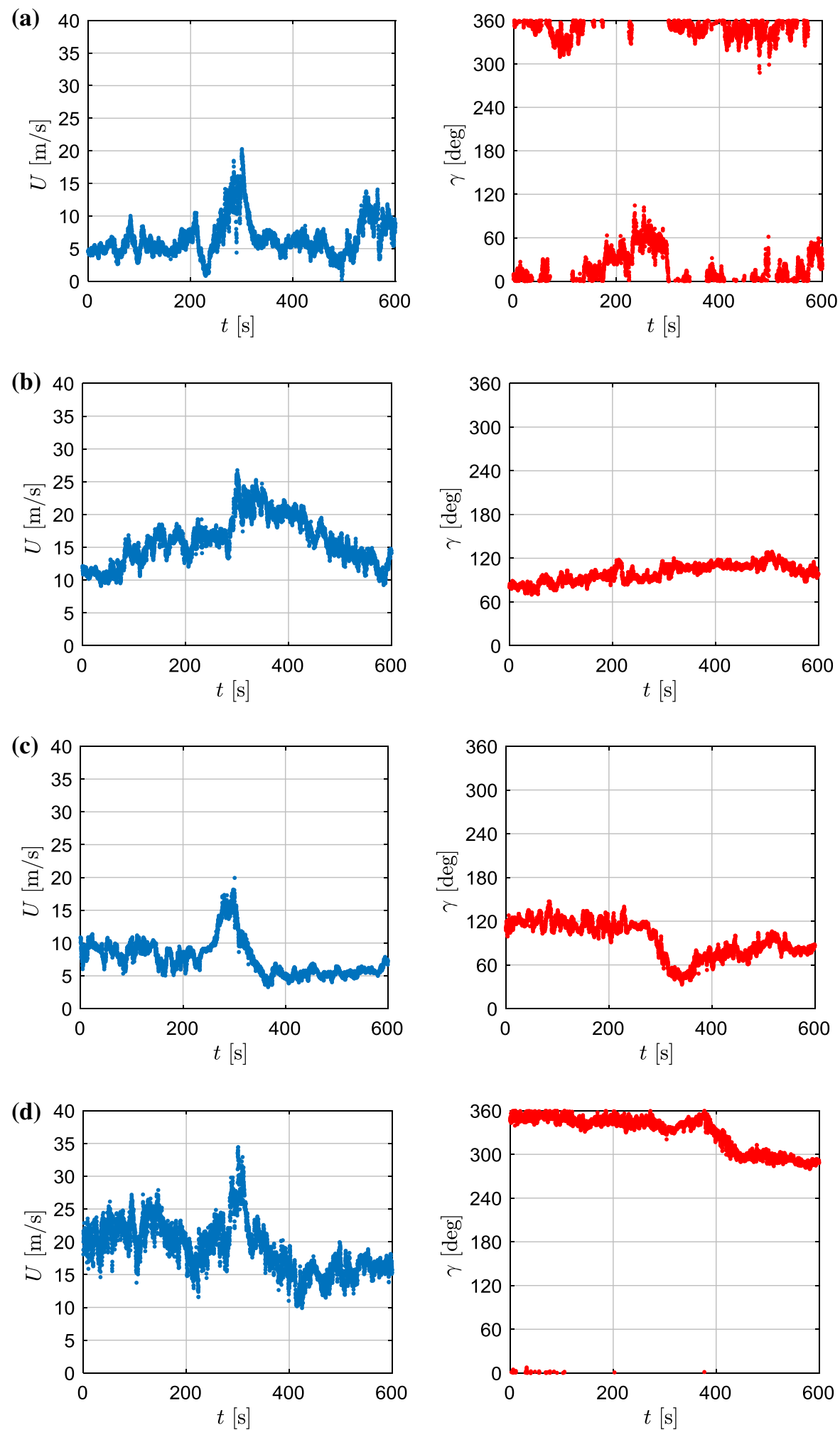

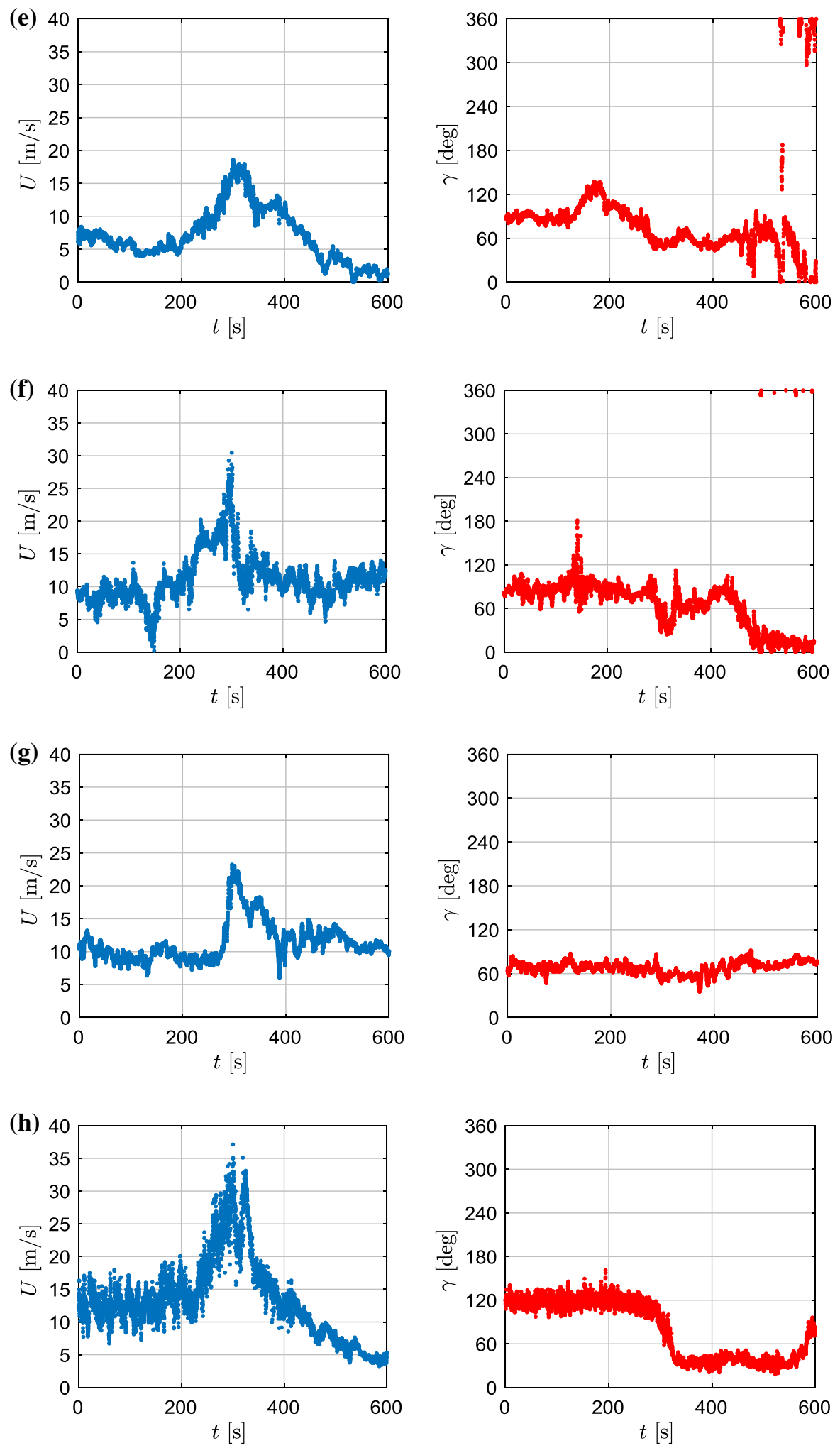
Fig. 22 continued
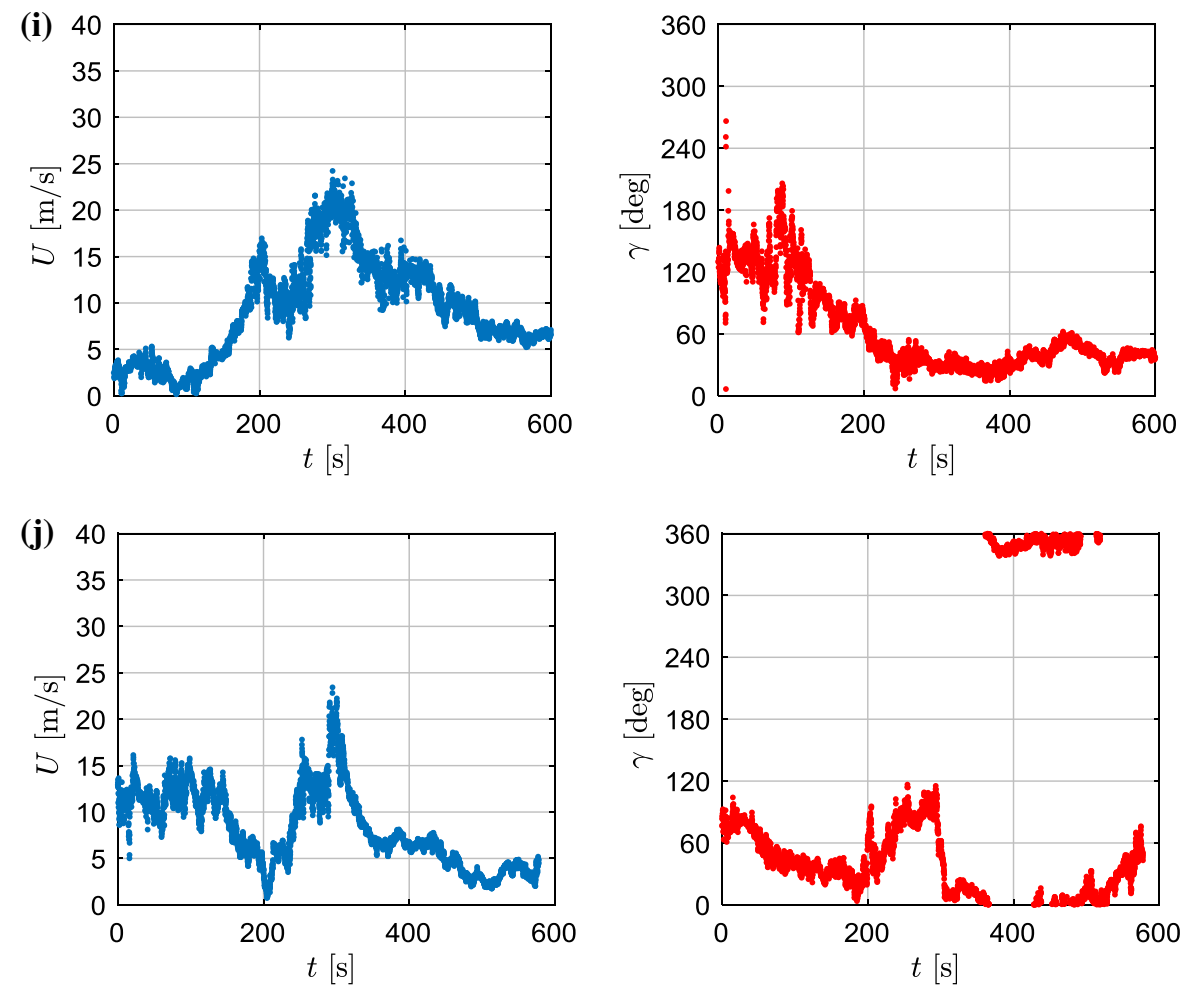

\section{References}

1. Van der Hoven I (1957) Power spectrum of horizontal wind speed in the frequency range from 0.0007 to 900 cycles per hour. J Meteorol 14:160-164

2. Davenport AG (1961) The application of statistical concepts to the wind loading of structures. Proc Inst Civ Eng 19(4):449-472

3. Piccardo G, Solari G (2000) 3-D wind-excited response of slender structures: closed form solution. J Struct Eng ASCE 126:936-943

4. Letchford CW, Mans C, Chay MT (2002) Thunderstormstheir importance in wind engineering (a case for the next generation wind tunnel). J Wind Eng Ind Aerod 90:1415-1433

5. Solari G (2014) Emerging issues and new frameworks for wind loading on structures in mixed climates. Wind Struct 19:295-320

6. Chen L, Letchford CW (2004) A deterministic-stochastic hybrid model of downbursts and its impact on a cantilevered structure. Eng Struct 26:619-629

7. Holmes JD, Hangan HM, Schroeder JL, Letchford CW, Orwig KD (2008) A forensic study of the Lubbock-Reese downdraft of 2002. Wind Struct 11:19-39

8. Xu YL, Chen J (2004) Characterizing nonstationary wind speed using empirical mode decomposition. J Struct Eng ASCE 130:912-920
9. Chen L, Letchford CW (2007) Numerical simulation of extreme winds from thunderstorm downbursts. J Wind Eng Ind Aerodyn 95:977-990

10. McCollough M, Kwon DK, Kareem A, Wang L (2014) Efficacy of averaging interval for nonstationary winds. J Eng Mech ASCE 140:1-19

11. Su Y, Huang G, Xu Y (2015) Derivation of time-varying mean for non-stationary downburst winds. J Wind Eng Ind Aerodyn 141:39-48

12. Choi ECC, Hidayat FA (2002) Dynamic response of structures to thunderstorm winds. Prog Struct Eng Mater 4:408-416

13. Solari G, Burlando M, De Gaetano P, Repetto MP (2015) Characteristics of thunderstorms relevant to the wind loading of structures. Wind Struct 20:763-791

14. Choi ECC (2000) Wind characteristics of tropical thunderstorms. J Wind Eng Ind Aerodyn 84:215-226

15. De Gaetano P, Repetto MP, Repetto T, Solari G (2014) Separation and classification of extreme wind events from anemometric records. J Wind Eng Ind Aerodyn 126:132-143

16. Chen L, Letchford CW (2004) Parametric study on the alongwind response of the CAARC building to downbursts in the time domain. J Wind Eng Ind Aerodyn 92:703-724

17. Huang G, Chen X, Liao H, Li M (2013) Predicting tall building response to nonstationary winds using multiple wind speed samples. Wind Struct 17:227-244

18. Le TH, Caracoglia L (2015) Reduced-order waveletGalerkin solution for the coupled, nonlinear stochastic 
response of slender buildings in transient winds. J Sound Vib 344:179-208

19. Solari G, Rainisio D, De Gaetano P (2017) Hybrid simulation of thunderstorm outflows and wind-excited response of structures. Meccanica 52:3197-3220

20. Solari G, De Gaetano P (2018) Dynamic response of structures to thunderstorm outflows: response spectrum technique vs time-domain analysis. Eng Struct 176:188-207

21. Chen X (2008) Analysis of alongwind tall building response to transient nonstationary winds. J Struct Eng ASCE 134:782-791

22. Kwon DK, Kareem A (2009) Gust-front factor: new framework for wind load effects on structures. J Struct Eng ASCE 135:717-732

23. Peng L, Huang G, Chen X, Yang Q (2018) Evolutionary spectra-based time-varying coherence function and application in structural response analysis to downburst winds. J Struct Eng ASCE 144:04018078

24. Solari G, De Gaetano P, Repetto MP (2015) Thunderstorm response spectrum: fundamentals and case study. J Wind Eng Ind Aerodyn 143:62-77

25. Solari G (2016) Thunderstorm response spectrum technique: theory and applications. Eng Struct 108:28-46

26. Le TH, Caracoglia L (2017) Computer-based model for the transient dynamics of a tall building during digitally simulated Andrews AFB thunderstorm. Comp Struct 193:44-72

27. Zhang S, Solari G, Burlando M, Yang Q (2019) Directional decomposition and analysis of thunderstorm outflows. J Wind Eng Ind Aerodyn 189:71-90

28. Solari G, Repetto MP, Burlando M, De Gaetano P, Pizzo M, Tizzi M, Parodi M (2012) The wind forecast for safety and management of port areas. $\mathrm{J}$ Wind Eng Ind Aerod 104-106:266-277

29. Repetto MP, Burlando M, Solari G, De Gaetano P, Pizzo M, Tizzi M (2018) A web-based GIS platform for the safe management and risk assessment of complex structural and infrastructural systems exposed to wind. Adv Eng Softw 117:29-45

30. Solari G (1988) Equivalent wind spectrum technique: theory and applications. J Struct Eng ASCE 114:1303-1323

31. Piccardo G, Solari G (1998) Generalized equivalent spectrum technique. Wind Struct 1:161-174

32. Solari G (2013) Brâncuşi Endless Column: a masterpiece of art and engineering. International Journal of High-Rise Buildings 2:193-212

33. CNR-DT 207/2008 (2009) Instructions for assessing wind actions and effects on structures. Rome, Italy

34. Wood GS, Kwok KCS (1998) An empirically derived estimate for the mean velocity profile of a thunderstorm downburst. In: Proceeding of the 7th Australian wind engineering society workshop, Auckland, New Zealand
35. Zhang S, Solari G, De Gaetano P, Burlando M, Repetto MP (2017) A refined analysis of thunderstorm outflow characteristics relevant to the wind loading of structures. Probab Eng Mech 54:9-24

36. Aboshosha H, Bitsuamlak G, El Damatty A (2015) Turbulence characterization of downbursts using LES. J Wind Eng Ind Aerod 136:44-61

37. Jubayer C, Elatar A, Hangan H (2016) Pressure distributions on a low-rise building in a laboratory simulated downburst. In: 8th International Colloquium on Bluff-Body Aerodynamics and Applications, Boston, MA

38. Lombardo FT, Smith DA, Schroeder JL, Mehta KC (2014) Thunderstorm characteristics of importance to wind engineering. J Wind Eng Ind Aerod 125:121-132

39. Peng L, Huang G, Chen X, Yang Q (2018) Evolutionary spectra-based time-varying coherence function and application in structural response analysis to downburst winds. J Struct Eng-ASCE 144:04018078

40. Solari G, Piccardo G (2001) Probabilistic 3-D turbulence modeling for gust buffeting of structures. Probab Eng Mech 16:73-86

41. Kawai H (1984) Pressure fluctuations on square prismsapplicability of strip and quasi-steady theories. J Wind Eng Ind Aerodyn 13:197-208

42. Currie IG, Turnbull DH (1987) Streamwise oscillations of cylinders near the critical Reynolds number. J Fluids Struct $1: 185-196$

43. Mason MS, Yang T, George T, Paxton O, Wong E (2016) An experimental investigation of the unsteady pressures on square cylinders in a non-stationary wind field. In: Proceedings of 8th International Colloquim on Bluff-Body Aerodynamics and Applications, Boston, MA

44. Carassale L, Freda A, Marrè-Brunenghi M (2013) Effetcs of free-stream turbulence and corner shape on the galloping instability of square cylinder. J Wind Eng Ind Aerod 123:274-280

45. Soize C (1978) Gust loading factors with nonlinear pressure terms. J Struct Div ASCE 104:991-1007

46. Kwon DK, Kareem A (2011) Peak factors for non-Gaussian load effects revisited. J Struct Eng ASCE 137:1611-1619

47. Davenport AG (1964) Note on the distribution of the largest value of a random function with application to gust loading. Proc Inst Civ Eng London 24:187-196

48. Piccardo G, Poggi S, Solari G (2018) Some critical issues on the distribution of the maximum value of the wind-excited response of structures. Prob Eng Mech 54:65-81

Publisher's Note Springer Nature remains neutral with regard to jurisdictional claims in published maps and institutional affiliations. 